\title{
Teoria Espectral para Semigrupos
}

\author{
Maria do Carmo Carbinatto
}

\author{
Orientação \\ Prof. Dr. José Gaspar Ruas Filho
}

Dissertação apresentada ao Instituto de Ciências Matemáticas de São Carlos USP, como parte dos requisitos para obtençâo do título de Mestre em Matemática.

USP - São Carlos

maio 1991 
Aos meus pais,

Celso e Angela.

Aos meus irmãos,

Luis, Rodinei e Edilaine. 
Não conto gozar a minha vida; nem em gozá-la penso. Só quero torná-la grande, ainda que para isso tenha de ser o meu corpo e a (minha alma) a lenha desse fogo. 
Primeiramente, agradeço a Deus pela ajuda e força espiritual que pude encontrar nos momentos de necessidade; agradeço ao Gaspar por sua orientação segura e precisa, por sua dedicação, incentivo e amizade; aos meus pais, pela compreensão, dedicação e apoio recebidos e pelos esforços feitos para que eu conseguisse concluir mais uma etapa de minha vida; aos meus irmãos, pela amizade que nos une; aos docentes e técnicos do ICMSC-USP, pelo apoio recebido durante esses anos de mestrado; ao amigo Kasuo, pela amizade e ajuda nos problemas de edição deste trabalho; a todos os amigos da pós-graduação que sempre me deram apoio, amizade; ao $\mathrm{CNPq}$ pelo auxílio financeiro parcial recebido; e a todos que direta e indiretamente contribuíram para que esse trabalho fosse realizado. 


\begin{abstract}
The purpose of this work is to study the spectral theory for semigroups of bounded linear operators. A motivation for this study is the geometric theory of differential equations, where the spectral theory has fundamental importance. One of questions that interests us most is under which conditions the spectrum of semigroups can be obtained from the spectrum infinitesimal generator.
\end{abstract}




\section{Resumo}

O próposito deste trabalho é estudar a teoria espectral para semigrupos de operadores lineares limitados. Uma motivação para este estudo é a teoria geométrica de equações diferenciais, onde a teoria espectral tem importância fundamental. Uma das questões que nos interessa é em que condições o espectro do semigrupo pode ser obtido a partir do espectro de seu gerador infinitesimal. 


\section{Conteúdo}

Introdução

1 Semigrupos 1

1.1 Semigrupo Fortemente Contínuo de Operadores Lineares . . . . 1

1.2 Problema Abstrato de Cauchy . . . . . . . . . . . 5 5

1.3 Espectro e Resolvente de um Operador . . . . . . . . . . 7

1.4 Teoria Espectral para Semigrupos . . . . . . . . . . . 11

2 Álgebra de Banach $\quad 17$

2.1 Álgebra ............................. 17

2.2 Álgebras de Banach . . . . . . . . . . . . . . . 19

2.3 Teoria Espectral para Álgebras de Banach . . . . . . . . . . . 21

2.4 Teoria de Representação de Gelfand . . . . . . . . . . . . . 24

.2.5 Topologia de Gelfand . . . . . . . . . . . . . . . 29

2.6 Teoria Espectral para Semigrupos Eventualmente Contínuos . . 31

3 : Teoria Espectral para Espaços de Hilbert 38

3.1 Soluções Periódicas . . . . . . . . . . . . . . . 39

3.2 Um exemplo: O Semigrupo das Integrais Fracionárias $\ldots . . .43$ 
3.3 Um contra exemplo em Espaços de Banach . . . . . . . . . . . 59

$\begin{array}{ll}\text { Bibliografia } & 63\end{array}$ 


\section{Introdução}

O objetivo geral deste trabalho é estudar a teoria espectral para semigrupos de operadores lineares limitados. Uma motivação para este estudo é obtido quando estudamos a teoria geométrica de equações diferenciais onde a teoria espectral tem importância fundamental. Uma das propriedades que interessa é em que condições o espectro do semigrupo $T(t)$ pode ser obtido a partir do espectro do gerador infinitesimal $A$. Quando $A$ é um operador linear limitado vale o seguinte

Teorema Espectral: Seja $T(t), t \geq 0$, um semigrupo de operadores lineares irnitados uniformemente contínuo e $A$ seu gerador infinitesimal. Então

$$
\sigma(T(t))=e^{t \sigma(A)} .
$$

Quando $A$ não é limitado o teorema espectral acima, em geral, não é mais válido. Estamos interessados em saber se existe alguma classe de semigrupos onde ainda é possível conseguir o teorema espectral e para aqueles em que deixa de ser vảlido determinar quais partes do espectro continuam satisfazendo o teorema acima.

No Capítulo 1 apresentamos as noções de semigrupos de operadores lineares limitados, problema abstrato de Cauchy para operadores lineares fechados, espectro e resolvente de um operador linear definido sobre um espaço de Banach. Encerramos o capítulo analisando quais as partes do espectro continuam satisfazendo o teorema espectral no caso de geradores não limitados em espaços de Banach.

Iniciamos o Capítulo 2 com um estudo sobre álgebra complexa de Banach. Mostramos que é possível introduzir o conceito de espectro e resolvente para um elemento de uma álgebra de Banach, de maneira a estender o conceito de espectro e resolvente introduzidos no Capítulo 1 para os elementos da álgebra dos operadores lineares limitados, $L(X)$. O Teorema (2.7) mostra que para álgebras de 
Banach é possível obter um teorema espectral para polinômios. O resultado central da teoria de álgebras de Banach é o Teorema de Representação de Gelfand. Utilizando esta representação obtemos uma caracterização do espectro de um elemento de uma álgebra complexa de Banach. Com esta caracterização determinamos uma classe de semigrupos definidos sobre um espaço de Banach onde o teorema espectral continua válido.

$\mathrm{O}$ último Capítulo tem por objetivo fazer um estudo dos semigrupos de operadores lineares definidos sobre um espaço de Hilbert. O Teorema (3.3) nos mostra que é possivel determinar condições sobre o resolvente do gerador infinitesimal de um semigrupo de modo a nos permitir concluir quais pontos do conjunto resolvente do gerador infinitesimal do semigrupo dão origem a pontos no espectro do semigrupo. Devemos ressaltar que este resultado dá condições sobre o gerador infinitesimal $A$ e seu operador resolvente e não sobre o semigrupo como no Capitulo 2. Apresentamos, em seguida, dois exemplos. 0 primeiro exemplo trata de integrais fracionárias definidas sobre $L_{2}(0,1)$. A relação entre o espectro do semigrupo e do seu resolvente fica completamente entendida com o Teorema (3.3). O segundo exemplo mostra que o Teorema (3.2) não é verdadeiro em espaços de Banach. 


\section{Capítulo 1}

\section{Semigrupos}

Neste capítulo desenvolvemos resultados básicos para o estudo da teoria espectral para semigrupos de operadores lineares. Na Seção (1.1) apresentamos definições e resultados simples da teoria geral de semigrupos. Na Seção (1.2) introduzimos o conceito de Problema Abstrato de Cauchy que será usado no Capítulo 3. Nas Seções (1.3) e (1.4) introduzimos várias noções de espectro e apresentamos os primeiros teoremas que relacionam o espectro de um semigrupo com o espectro de seu gerador infinitesimal.

\subsection{Semigrupo Fortemente Contínuo de Ope- radores Lineares}

Definição 1.1 Seja $X$ um espaço de Banach. Uma famalia $T(t), t \geq 0$, de operadores lineares limitados de $X$ em $X$ é um semigrupo de operadores lineares limitados sobre $X$ se

1. $T(0)=I$.

2. $T(t+s)=T(t) T(s)$ para todo $t, s \geq 0$. Além disso,

3. se $\lim _{t \rightarrow 0^{+}}\|T(t)-I\|=0$, então $T(t)$ é um semigrupo uniformemente contínuo. 
4. se $\lim _{t \rightarrow 0^{+}} T(t) x=x$ para todo $x \in X$, dizemos que $T(t)$ é um semigrupo fortemente contínuo ou um $C_{0}$-semigrupo.

Definição 1.2 Seja $T(t)$ um $C_{0}$-semigrupo sobre um espaço de Banach $X$. $O$ semigrupo $T(t)$ é chamado

1. eventualmente contínuo se existe $t_{0}>0$, tal que $t \rightarrow T(t)$ é contínuo para $t>t_{0}$.

2. eventualmente diferenciável se existe $t_{0}>0$, tal que para todo $x \in X$, $t \rightarrow T(t) x$ é diferenciável para $t>t_{0}$.

3. eventualmente compacto se existe $t_{0}>0$, tal que $T(t)$ é operador compacto para $t>t_{0}$.

Definição 1.3 Seja $\Delta=\left\{z: \varphi_{1}<\arg z<\varphi_{2}, \quad\right.$ varphi $\left.i_{1}<0<\varphi_{2}\right\}$. Para $z \in \Delta$, seja $T(z)$ um operador linear limitado. A familia $T(z), z \in \Delta$, é um semigrupo analítico em $\Delta$ se

1. $z \rightarrow T(z)$ é analítica em $\Delta$.

2. $T(0)=I$ e $\lim _{z \rightarrow 0} T(z) x=x, z \in \Delta$, para todo $x \in X$.

3. $T\left(z_{1}+z_{2}\right)=T\left(z_{1}\right) T\left(z_{2}\right)$, para $z_{1}, z_{2} \in \Delta$.

Definição 1.4 Seja $T(t), t \geq 0$, um semigrupo. $O$ operador linear $A$ definido por

$$
A x=\lim _{t \rightarrow 0^{+}} \frac{T(t) x-x}{t} \text { para } x \in \mathcal{D}(A)
$$

onde

$$
\mathcal{D}(A)=\left\{x \in X: \lim _{t \rightarrow 0^{+}} \frac{T(t) x-x}{t} \text { existe }\right\},
$$

é chamado o gerador infinitesimal do semigrupo $T(t)$, e $\mathcal{D}(A)$ é o dominio de $A$.

Teorema 1.1 Seja $T(t)$ um $C_{0}$-semigrupo. Existem $\omega \in \mathbf{R}$ e $M \geq 1$ tais que $\|T(t)\| \leq M e^{\omega t}, t \geq 0$. 
Prova- Existe $\eta$ tal que $\|T(t)\|$ é limitado em $[0, \eta]$. Caso contrário, existe uma sequência $\left(t_{n}\right)$, satisfazendo $t_{n} \geq 0, \lim _{n \rightarrow \infty} t_{n}=0 \mathrm{e}\left\|T\left(t_{n}\right)\right\| \geq n$. Do Princípio da Limitação Uniforme, existe $x \in X$ tal que $\left\|T\left(t_{n}\right) x\right\|$ não é limitado. Mas isso contradiz o fato de $T(t)$ ser um $C_{0}$-semigrupo. Portanto, $\|T(t)\| \leq K$ para $0 \leq t \leq \eta$. Ainda, $K \geq 1$. Tomando $\omega \geq \eta^{-1} \log \|T(\eta)\|$, temos que dado $t \geq 0$, $t=n \eta+\delta$ com $0 \leq \delta \leq \eta \mathrm{e}$

$$
\|T(t)\| \leq\|T(\eta)\|^{n}\|T(\delta)\| \leq M e^{\omega t}
$$

onde $M=K e^{|\omega| \eta}$.

Teorema 1.2 Sejam $T(t)$ um $C_{0}$-semigrupo e A seu gerador infinitesimal.

1. Para todo $x \in X, t \in[0, \infty) \rightarrow T(t) x \in X$ é contínua.

2. Se $x \in X$, então

$$
\lim _{h \rightarrow 0} \frac{1}{h} \int_{t}^{t+h} T(s) x d s=T(t) x .
$$

3. Se $x \in X$, então $\int_{0}^{t} T(s) x d s \in \mathcal{D}(A) e$

$$
A \int_{0}^{t} T(s) x d s=T(t) x-x .
$$

4. $T(t) \mathcal{D}(A) \subset \mathcal{D}(A)$ e se $x \in \mathcal{D}(A)$ então $t \in[0, \infty) \rightarrow T(t) x \in \mathcal{D}(A)$ é diferenciável $e$

$$
\frac{d}{d t} T(t) x=A T(t) x=T(t) A x .
$$

5. Se $x \in \mathcal{D}(A)$, então

$$
T(t) x-T(s) x=\int_{s}^{t} T(\tau) A x d \tau=\int_{s}^{t} A T(\tau) x d \tau .
$$

Prova- A afirmativa 1 segue das desigualdades

$$
\begin{aligned}
& \|T(t+h) x-T(t) x\| \leq\|T(t)\|\|T(h) x-x\| \text { para } t, h \geq 0, \\
& \|T(t-h) x-T(t) x\| \leq\|T(t-h)\|\|x-T(h) x\| \text { para } t \geq h \geq 0
\end{aligned}
$$

e do Teorema (1.1). A afirmativa 2 segue diretamente de 1. Para mostrar 3 seja $x \in X$ e $h>0$. Temos

$$
\frac{T(h)-I}{h} \int_{0}^{1} T(s) x d s=\frac{1}{h} \int_{t}^{t+h} T(s) x d s-\frac{1}{h} \int_{0}^{h} T(s) x d s
$$


e quando $h \rightarrow 0$ o lado direito tende para $T(t) x-x$. Para a afirmativa 4 seja $x \in \mathcal{D}(A)$ e $h>0$. Assim,

$$
\frac{T(h)-I}{h} T(t) x=T(t)\left(\frac{T(h)-I}{h}\right) \rightarrow T(t) A x \text { quando } h \rightarrow 0 .
$$

Logo, $T(t) x \in \mathcal{D}(A)$ e $A T(t) x=T(t) A x$. Resta provarmos que para $t>0$, a derivada à esquerda de $T(t) x$ existe e é igual a $T(t) A x$. Isto segue da igualdade

$$
\begin{aligned}
\frac{T(t-h) x-T(t) x}{-h}-T(t) A x & =T(t-h)\left(\frac{T(h) x-x}{h}-A x\right) \\
& +(T(t-h) A x-T(t) A x) .
\end{aligned}
$$

A parte 5 é obtida por integração de $s$ a $t$ da igualdade encontrada em 4 .

Corolário 1.1 Se $A$ é o gerador infinitesimal de um $C_{0}$-semigrupo $T(t)$, então

1. A é um operador linear fechado densamente definido.

2. se $\Re(\lambda)>\omega, \rho(A)$ denota o conjunto de resolvente do operador $A$ e $R(\lambda, A)=$ $(\lambda I-A)^{-1}$, o operador resolvente de $A$, então $\lambda \in \rho(A), R(\lambda, A) x=$ $\int_{0}^{\infty} e^{-\lambda t} T(t) x d t$ e $\|R(\lambda, A)\| \leq M /(\Re(\lambda)-\omega)$.

Prova- Para todo $x \in X$ seja $x_{t}=1 / t \int_{0}^{t} T(s) x d s$. Logo, $x_{t} \in \mathcal{D}(A)$ e $x_{t} \rightarrow x$ quando $t \rightarrow 0^{+}$. Assim, $\overline{\mathcal{D}(A)}=X$. Para provarmos que $A$ é um operador linear fechado, seja $x_{n} \in \mathcal{D}(A)$ tal que $x_{n} \rightarrow x$ e $A x_{n} \rightarrow y$ quando $n \rightarrow \infty$. Da parte 4 do Teorema (1.2) temos que

$$
T(t) x_{n}-x_{n}=\int_{0}^{t} T(s) A x_{n} d s .
$$

Como o integrando do lado direito da igualdade acima converge uniformemente para $T(s) y$ em intervalos limitados, temos

$$
T(t) x-x=\int_{0}^{t} T(s) y d s .
$$

Portanto,

$$
\lim _{t \rightarrow 0^{+}} \frac{T(t) x-x}{t}=y
$$

Ousseja, $x \in \mathcal{D}(A)$ e $A x=y$.

Para a segunda parte, seja $\Re(\lambda)>\omega$. Definamos

$$
R(\lambda) x=\int_{0}^{\infty} e^{-\lambda t} T(t) x d t
$$


Temos $\|R(\lambda) x\| \leq M\|x\| /(\Re(\lambda)-\omega)$. Se $x \in X$ e $h>0$, então

$$
\frac{T(h)-I}{h} R(\lambda) x=\frac{e^{\lambda h-1}}{h} \int_{0}^{\infty} e^{-\lambda t} T(t) x d t-\frac{e^{\lambda h}}{h} \int_{0}^{h} e^{-\lambda t} T(t) x d t \rightarrow \lambda R(\lambda) x-x
$$

quando $h \rightarrow 0$. Ou seja, $A R(\lambda) x=\lambda R(\lambda) x-x$.

Seja $x \in \mathcal{D}(A)$. Sendo $A$ um operador fechado temos

$$
R(\lambda) A x=A \int_{0}^{\infty} e^{-\lambda t} T(t) x d t=\lambda R(\lambda) x-x .
$$

Portanto, $\lambda \in \rho(A)$.

\subsection{Problema Abstrato de Cauchy}

Sejam $X$ um espaço de Banach e $A: \mathcal{D}(A) \subset X \rightarrow X$ um operador linear. Dado $x \in X$ o problema abstrato de Cauchy para $A$, com condição inicial $x$, consiste em determinar a solução $u(t)$ do problema de valor inicial

$$
\left\{\begin{array}{l}
\dot{u}(t)=A u(t), t>0 \\
u(0)=x
\end{array} .\right.
$$

Definição 1.5 Uma função $u:[0, \infty) \rightarrow X$ é uma solução (clássica ou estrita) se

1. $u(t)$ é contínua para $t \geq 0$.

2. $u(t)$ é continuamente diferenciável e $u(t) \in \mathcal{D}(A)$ para $t>0$.

3. (1.1) está satisfeita.

No caso em que $A$ é o gerador infinitesimal de um $C_{0}$-semigrupo $T(t)$, o problema abstrato de Cauchy para $A$ tem uma solução, a saber, $u(t)=T(t) x$ para todo $x \in \mathcal{D}(A)$. Além disso, a solução é única. 0 teorema a seguir determina condições necessárias e suficientes para que o problema do valor inicial (1.1) possua uma única solução para $x \in \mathcal{D}(A)$, cuja demonstração pode ser encontrada em Pazy [9, pag. 102]. 
Teorema 1.3 Seja $A$ um operador linear densamente definido tal que $\rho(A) \neq \emptyset$. O problema do valor inicial (1.1) tem uma única solução para todo $x \in \mathcal{D}(A)$, que é diferenciável em $[0, \infty)$ se, e somente se, $A$ é o gerador infinitesimal de um $C_{0}$-semigrupo $T(t)$.

Consideremos o problema do valor inicial não homogêneo

$$
\left\{\begin{array}{l}
\dot{u}(t)=A u(t)+f(t), \quad t>T \\
u(0)=x
\end{array},\right.
$$

onde $f:[0, T) \rightarrow X$ e $A$ é o gerador infinitesimal de um $C_{0}$-semigrupo $T(t)$.

Definição 1.6 Uma função $u:[0, T) \rightarrow X$ é uma solução (clássica ou estrita) se

1. $u(t)$ é contínua para $t \in[0, T)$.

2. $u(t)$ é continuamente diferenciável sobre $(0, T)$ e $u(t) \in \mathcal{D}(A)$ para $0<t<T$.

3. (1.2) está satisfeita.

Corolário 1.2 Se $f \in L_{1}([0, T], X)$, então para todo $x \in X$, o problema do valor inicial (1.2) tem no máximo uma solução. Se (1.2) tem uma solução, então é dada por

$$
u(t)=T(t) x+\int_{0}^{t} T(t-s) f(s) d s
$$

Prova- Seja $u(t)$ uma solução de (1.2) e seja $g(s)=T(t-s) u(s)$. Então $g$ é diferenciável para $0<s<t$ e

$$
\frac{d g}{d s}=-A T(t-s) u(s)+T(t-s)[A u(s)+f(s)]=T(t-s) f(s) .
$$

Integrando entre 0 e $t$ temos

$$
u(t)=T(t) u(0)+\int_{0}^{t} T(t-s) f(s) d s .
$$

Definição 1.7 função $u \in C([0, T], X)$, definida por

$$
u(t)=T(t) x+\int_{0}^{t} T(t-s) f(s) d s
$$

é chamada solução fraca de (1.2). 
A demonstração do Teorema abaixo e seu corolário podem ser encontradas em Pazy [9, pag. 107].

Teorema 1.4 Seja $A$ o gerador infinitesimal de um $C_{0}$-semigrupo $T(t)$. Sejam $f \in L_{1}([0, T], X)$, contínua em $(0, T]$ e $v(t)=\int_{0}^{t} T(t-s) f(s) d s$ com $t \in[0, T]$. O problema do valor inicial (1.2) tem uma solução u em $[0, T)$, para todo $x \in \mathcal{D}(A)$, se uma das hipóteses abaixo é verificada:

1. $v(t)$ é continuamente diferenciável em $(0, T)$.

2. $v(t) \in \mathcal{D}(A)$ para $0<t<T$ e $A v(t)$ é contínua sobre $(0, T)$.

Se (1.2) tem uma solução em $[0, T)$ para algum $x \in \mathcal{D}(A)$ então as duas condições acima estão verificadas.

Corolário 1.3 Seja A o gerador infinitesimal de um $C_{0}$-semigrupo $T(t)$. Se uma das hipóteses abaixo é verificada:

1. $f$ é continuamente diferenciável em $[0, T]$

2. $f \in L_{1}([0, T], X)$ é contínua em $(0, T), f(s) \in \mathcal{D}(A)$ para $0<s<t e$ $A f(s) \in L_{1}([0, T], X)$

então o problema do valor inicial (1.2) tem uma única solução para cada $x \in$ $\mathcal{D}(A)$.

\subsection{Espectro e Resolvente de um Operador}

Definição 1.8 Seja A um operador linear definido em um espaço de Banach $X$. Dizemos que

1. $\lambda \in I_{3}(A)$, se $\lambda I-A$ não é injetor e $R(\lambda I-A)=X$.

2. $\lambda \in I I_{3}(A)$, se $\lambda I-A$ não é injetor e $\overline{R(\lambda I-A)}=X$ mas $R(\lambda I-A) \neq X$.

3. $\lambda \in I I I_{3}(A)$, se $\lambda I-A$ não é injetor e $\overline{R(\lambda I-A)} \neq X$. 
4. $\lambda \in I I I_{1}(A)$, se $\lambda I-A$ é injetor, $(\lambda I-A)^{-1}$ é limitado e $R(\lambda I-A) \neq X$.

5. $\frac{\lambda \in I I I_{2}}{R(\lambda I-A)}$, se $\lambda I-A$ é injetor, $(\lambda I-A)^{-1}$ não é limitado $e$ $\overline{R(\lambda I-A)} \neq X$.

6. $\lambda \in I_{2}(A)$, se $\lambda I-A$ é injetor, $(\lambda I-A)^{-1}$ não é limitado $e$ $R(\lambda I-A)=X$.

7. $\lambda \in I I_{2}(A)$, se $\lambda I-A$ é injetor, $(\lambda I-A)^{-1}$ não é limitado e $\overline{R(\lambda I-A)}=X$ mas $R(\lambda I-A) \neq X$.

8. $\lambda \in I_{1}(A)$, se $\lambda I-A$ é injetor, $(\lambda I-A)^{-1}$ é limitado e $R(\lambda I-A)=X$.

9. $\lambda \in I I_{1}(A)$, se $\lambda I-A$ é injetor, $(\lambda I-A)^{-1}$ é limitado e $\overline{R(\lambda I-A)}=X$ mas $R(\lambda I-A) \neq X$.

Definição 1.9 Seja $A$ um operador linear definido em um espaço de Banach $X$. Dado $\lambda \in \mathbf{C}$ dizemos que

1. $\lambda$ é um elemento do espectro pontual de $A, P \sigma(A)$, se $\lambda I-A$ não é injetor;

2. $\lambda$ é um elemento do espectro residual de $A, R \sigma(A)$, se $\lambda I-A$ é injetor $e$ $\overline{R(\lambda I-A)} \neq X$

3. $\lambda$ é um elemento do espectro contínuo de $A, C \sigma(A)$, se $\lambda I-A$ é injetor, $\overline{R(\lambda I-A)}=X$ mas $(\lambda I-A)^{-1}$ náo é um operador linear limitado.

$A$ união destes conjuntos forma o espectro de $A, \sigma(A)$. O seu complementar é chamado resolvente de $A, \rho(A)$, ou seja, $\lambda$ está no resolvente de $A$ se $\lambda I-A$ é injetor, $R(\lambda I-A)$ é um conjunto denso em $X$ e $(\lambda I-A)^{-1}$ é um operador linear limitado.

Segue imediatamente das definições acima que

1. $P \sigma(A)=I_{3}(A) \cup I I_{3}(A) \cup I I I_{3}(A)$.

2. $R \sigma(A)=I I I_{1}(A) \cup I I I_{2}(A)$.

3. $C \sigma(A)=I_{2}(A) \cup I I_{2}(A)$. 
4. $\rho(A)=I_{1}(A) \cup I I_{1}(A)$.

Um dos problemas mais importantes na teoria de semigrupos é o comportamento assintótico de um semigrupo. Para o conhecimento deste comportamento é necessário informações sobre o espectro, $\sigma(T(t))$, do semigrupo.

$O$ resultado a seguir nos mostra o que o conhecimento do espectro do semigrupo nos permite concluir.

Teorema 1.5 Seja $T(t), t \geq 0$, um $C_{0}$-semigrupo. Suponhamos que para algum $t_{0}>0$ e $\alpha \in \mathbf{R}, \sigma\left(T\left(t_{0}\right)\right) \cap\left\{\lambda:|\lambda|=e^{\alpha t_{0}}\right\}=0$. Então existe $E \in L(X)$, projeção (contínua) tal que $E T(t)=T(t) E$, para todo $t \geq 0$. Se $X_{+}=R(E)$ e $X_{-}=N(E)$ então $\left.T(t)\right|_{X_{+}}$e $\left.T(t)\right|_{X_{-}}$satisfazem:

1. $\left.T(t)\right|_{X_{+}} \in L\left(X_{+}\right)$e $\left.T(t)\right|_{X_{-}} \in L\left(X_{-}\right)$.

2. $\sigma\left(\left.T(t)\right|_{X_{+}}\right)=\sigma(T(t)) \cap\left\{\lambda:|\lambda|<e^{\alpha t}\right\}=\sigma_{t}^{+}$.

3. $\sigma\left(\left.T(t)\right|_{X_{-}}\right)=\sigma(T(t)) \cap\left\{\lambda:|\lambda|>e^{\alpha t}\right\}=\sigma_{t}^{-}$.

Além disso, existem constantes $M \geq 1$ e $\delta>0$ tais que

4. $\left\|\left.T(t)\right|_{X_{+}}\right\| \leq M e^{(\alpha-\delta) t}, t \geq 0$.

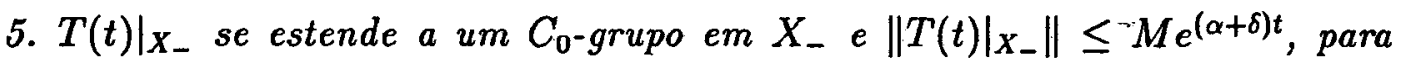
$t \leq 0$.

Prova- Seja

$$
E=\frac{1}{2 \pi i} \int_{|\lambda|=e^{\alpha t_{0}}} R\left(\lambda, T\left(t_{0}\right)\right) d \lambda .
$$

Temos $E \in L(X)$ e $E^{2}=E$. Como $T(t)$ comuta com $T\left(t_{0}\right)$ temos que $E$ comuta com $T(t)$. Sejam $X_{+}=R(E)$ e $X_{-}=N(E)$. É fácil ver que $T(t)\left(X_{+}\right) \subset X_{+}$e $T(t)\left(X_{-}\right) \subset X_{-}$. Portanto, temos provado a primeira afirmativa do teorema. Agora, $\sigma\left(T\left(t_{0}\right)\right)=\sigma_{t_{0}}^{+} \cup \sigma_{t_{0}}^{-}$onde

$$
\sigma_{t_{0}}^{+}=\sigma\left(T\left(t_{0}\right)\right) \cap\left\{\lambda:|\lambda|<e^{\alpha t_{0}}\right\}
$$

e

$$
\sigma_{t_{0}}^{-}=\sigma\left(T\left(t_{0}\right)\right) \cap\left\{\lambda:|\lambda|>e^{\alpha t_{0}}\right\} .
$$


Portanto, $\sigma\left(\left.T\left(t_{0}\right)\right|_{X_{+}}\right) \subset\left\{\lambda:|\lambda|<e^{\alpha t_{0}}\right\}$. Logo, $r\left(\left.T\left(t_{0}\right)\right|_{X_{+}}\right)<e^{\left(\alpha-\delta_{1}\right) t_{0}}$ onde $\delta_{1}=d / 2 \operatorname{com} d=\operatorname{dist}\left(\sigma\left(\left.T\left(t_{0}\right)\right|_{X_{+}}\right),\left\{\lambda:|\lambda|<e^{\alpha t_{0}}\right\}\right)$. Esta relação vale para todo $t \geq 0$. De fato, para cada $k \in \mathbf{N}$, temos $k t=t_{0} n_{k}+\tau_{k} \operatorname{com} 0 \leq \tau_{k} \leq t_{0}$. Ainda, $n_{k} \rightarrow \infty$ quando $k \rightarrow \infty$ e $\|T(t)\| \leq M$ para $0 \leq t<t_{0}$. Com isso

$$
\begin{aligned}
r\left(\left.T(t)\right|_{X_{+}}\right) & =\lim _{k \rightarrow \infty}\left\|\left(\left.T(t)\right|_{X_{+}}\right)^{k}\right\|^{\frac{1}{k}}=\lim _{k \rightarrow \infty}\left\|\left.T(k t)\right|_{X_{+}}\right\|^{\frac{1}{k}} \\
& \leq \lim _{k \rightarrow \infty}\left\|\left.T\left(n_{k} t_{0}\right)\right|_{X_{+}}\right\|^{\frac{t_{n} t_{0}+\tau_{k}}{k}}\left\|\left.T\left(\tau_{k}\right)\right|_{X_{+}}\right\|^{\frac{t}{n_{k} t_{0}+\tau_{k}}} \\
& \leq \lim _{k \rightarrow \infty}\left\|\left.T\left(n_{k} t_{0}\right)\right|_{X_{+}}\right\|^{\frac{t}{n_{k}\left(t_{0}+\tau_{k} / n_{k}\right)}} M^{\frac{1}{n_{k}\left(t_{0}+\tau_{k} / n_{k}\right)}} \\
& =r\left(\left.T\left(t_{0}\right)\right|_{X_{+}}\right)^{\frac{t}{t_{0}}}<e^{\left(\alpha-\delta_{1}\right) t}
\end{aligned}
$$

Portanto, $\sigma\left(\left.T(t)\right|_{X_{+}}\right) \subset \sigma_{t}^{+}$. Como $\sigma\left(\left.T\left(t_{0}\right)\right|_{X_{-}}\right) \subset\left\{\lambda:|\lambda|>e^{\alpha t_{0}}\right\}$, $0 \in \rho\left(\left.T\left(t_{0}\right)\right|_{X_{-}}\right)$. Assim, pelo Teorema (1.6.5) [9, pag. 24], temos que $\left.T(t)\right|_{X_{-}}$ pode ser imerso em um $C_{0}$-grupo e $\left.T(-t)\right|_{X_{-}}=\left(\left.T(t)\right|_{X_{-}}\right)^{-1}$ para $t \geq 0$. Além disso, $\left\{\lambda:|\lambda| \leq e^{\alpha t_{0}}\right\} \subset \rho\left(T\left(t_{0}\right) \mid x_{-}\right)$, e portanto, $\left\{\lambda:|\lambda| \geq e^{-\alpha t_{0}}\right\} \subset$ $\rho\left(\left(\left.T\left(t_{0}\right)\right|_{X_{-}}\right)^{-1}\right)$. Assim, existe $\delta_{2}$ tal que $r\left(\left.T\left(-t_{0}\right)\right|_{X_{-}}\right)<e^{-\left(\alpha+\delta_{2}\right) t_{0}}$.

Portanto, $r\left(\left.T(-t)\right|_{X_{-}}\right)<e^{-\left(\alpha+\delta_{2}\right) t}$ para $t \geq 0$. Desse modo, $\sigma\left(\left.T(t)\right|_{X_{-}}\right) \subset \sigma_{t}^{-}$. Podemos concluir que $\sigma(T(t))=\sigma_{t}^{+} \cup \sigma_{t}^{-} \mathrm{e}$

$$
\sigma\left(\left.T(t)\right|_{X_{+}}\right)=\sigma_{t}^{+} \text {e } \sigma\left(\left.T(t)\right|_{X_{-}}\right)=\sigma_{t}^{-} .
$$

O que prova as afirmativas 2 e 3 .

Para verificar 4 observemos que

$$
\left\|\left.T\left(n t_{0}\right)\right|_{X_{+}}\right\| \leq M_{0} e^{n\left(\alpha-\delta_{1}\right) t_{0}} \text { para } n \geq 0 .
$$

Tomando $t \geq 0$ temos $t=n t_{0}+\tau \mathrm{e}$

$$
\begin{aligned}
\left\|\left.T(t)\right|_{X_{+}}\right\| & =\left\|\left.T\left(n t_{0}+\tau\right)\right|_{X_{+}}\right\| \leq\left\|\left.T\left(n t_{0}\right)\right|_{X_{+}}\right\|\left\|\left.T(\tau)\right|_{X_{+}}\right\| \\
& \leq M_{0} \sup _{0 \leq \tau \leq t_{0}}\left[\left\|\left.T(\tau)\right|_{X_{+}}\right\| e^{-(\alpha-\delta) \tau}\right] e^{(\alpha-\delta)\left(n t_{0}+\tau\right)} \\
& =M e^{(\alpha-\delta) t}
\end{aligned}
$$

A primeira parte a afirmativa 5 já foi obtida acima. A segunda, segue de modo análogo à 4 .

Quando estamos estudando um problema de Cauchy conhecemos o gerador infinitesimal e não o semigrupo por ele gerado. Por isto é de fundamental importância conhecer as relações entre o espectro do semigrupo $T(t)$ e o espectro de seu gerador infinitesimal $\sigma(A)$.

Encontramos uma motivação para estas relações quando o gerador infinitesimal $A$ é um operador linear limitado. Para este caso temos que o semigrupo 
gerado por $A$ é $T(t)=e^{t A}$ e então a seguinte relação entre os espectros é válida:

$$
\sigma(T(t))=e^{t \sigma(A)}
$$

De modo mais preciso esta igualdade se traduz em:

1. se $\lambda \in \sigma(A)$ então $e^{\lambda t} \in \sigma(T(t))$.

2. se $e^{\lambda t} \in \sigma(T(t))$ então existe $n \in \mathbf{N}$ tal que $\lambda_{n}=\lambda+2 \pi i n / t \in \sigma(A)$.

Estamos interessados numa relação deste tipo para o caso em que $A$ não é limitado. Porém a igualdade, em geral não ocorre. Assim, nosso primeiro objetivo é verficar se pelos menos uma das inclusões acima é verdadeira e tentar saber qual parte do espectro é responsável pela falha da igualdade mencionada. O que será visto na próxima seção.

\subsection{Teoria Espectral para Semigrupos}

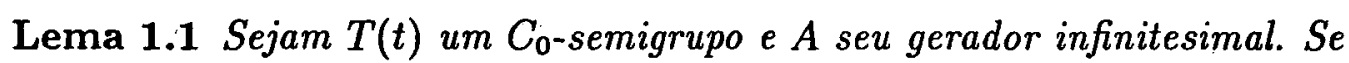

$$
B_{\lambda}(t) x=\int_{0}^{t} e^{\lambda(t-s)} T(s) x d s
$$

então

$$
\begin{gathered}
(\lambda I-A) B_{\lambda}(t) x=e^{\lambda t} x-T(t) x, \text { para todo } x \in X \\
B_{\lambda}(t)(\lambda I-A) x=e^{\lambda t} x-T(t) x, \text { para todo } x \in \mathcal{D}(A)
\end{gathered}
$$

Prova- É fácil ver que $B_{\lambda}(t)$ é um operador linear para cada $t \geq 0$. Ainda,

$$
\begin{aligned}
\left\|B_{\lambda}(t) x\right\| & \leq \int_{0}^{t} e^{\Re(\lambda)(t-s)}\|T(s)\|\|x\| d s \\
& \leq \frac{M}{|\Re(\lambda)-\omega|}\left|e^{-\omega t}-e^{\Re(\lambda) t}\right|\|x\|,
\end{aligned}
$$

o que mostra que $B_{\lambda}(t)$ é um operador linear limitado.

Como $B_{\lambda}(t)$ comuta com $A$ para $x \in \mathcal{D}(A)$ temos,

$$
B_{\lambda}(t)(\lambda I-A) x=(\lambda I-A) B_{\lambda}(t) x, \text { para todo } x \in \mathcal{D}(A) .
$$

Mostremos que $B_{\lambda}(t) x \in \mathcal{D}(A)$ para todo $x \in X$, ou seja,

$$
\lim _{h \rightarrow 0^{+}} \frac{T(h)-I}{h} B_{\lambda}(t) x \text { existe. }
$$


Desde que

$$
\begin{aligned}
\frac{T(h)-I}{h} B_{\lambda}(t) x & =\frac{1}{h}\left[\int_{0}^{t} e^{\lambda(t-s)} T(s+h) x d s-\int_{0}^{t} e^{\lambda(t-s)} T(s) x d s\right] \\
& =\frac{e^{\lambda h}-1}{h} \int_{h}^{t} e^{\lambda(t-s)} T(s) x d s+\frac{e^{\lambda h}}{h} \int_{t}^{t+h} e^{\lambda(t-s)} T(s) x d s \\
& -\frac{1}{h} \int_{0}^{h} e^{\lambda(t-s)} T(s) x d s,
\end{aligned}
$$

fazendo $h \rightarrow 0^{+}$, temos

$$
\lim _{h \rightarrow 0^{+}} \frac{T(h)-I}{h} B_{\lambda}(t) x=\lambda B_{\lambda}(t) x+T(t) x-e^{\lambda t} x .
$$

$\mathrm{O}$ resultado segue.

Teorema 1.6 Sejam $T(t)$ um $C_{0}$-semigrupo e A seu gerador infinitesimal. Então

$$
e^{\sigma(A) t} \subset \sigma(T(t)), t \geq 0 .
$$

Prova- Basta demonstrarmos a seguinte inclusão: $\rho(T(t)) \subset e^{p(A) t}$. Se $e^{\lambda t} \in \rho(T(t))$, então $\left(e^{\lambda t} I-T(t)\right)^{-1}$ existe e é limitado. Considerando $B_{\lambda}(t)$ como no Lema (1.1) temos que $T(t)$ comuta com $B_{\lambda}(t)$ e, conseqüentemente $\left(e^{\lambda t} I-T(t)\right)^{-1}$ comuta $B_{\lambda}(t)$. Logo,

$$
(\lambda I-A)\left(e^{\lambda t} I-T(t)\right)^{-1} B_{\lambda}(t) x=x, \text { para todo } x \in R\left(e^{\lambda t} I-T(t)\right)
$$

e

$$
\left(e^{\lambda t} I-T(t)\right)^{-1} B_{\lambda}(t)(\lambda I-A) x=x \text {, para todo } x \in \mathcal{D}(A) .
$$

E disto segue que $\lambda \in \rho(A)$.

No restante desta seção estudaremos as relações entre cada divisão do espectro (resp. resolvente) de $A$ e a correspondente do espectro (resp. resolvente) de $T(t)$.

No que segue, para $n \in \mathrm{N}, \lambda_{n}$ denotará $\lambda+2 n \pi i / t \operatorname{com} \lambda \in \mathrm{C}$ e $t \geq 0$.

Proposição 1.1 Sejam $T(t)$ um $C_{0}$-semigrupo e A seu gerador infinitesimal.

1. Se $\lambda \in I_{3}(A)$, então $e^{\lambda t} \in P \sigma(T(t))$.

2. Se $e^{\lambda t} \in I_{3}(T(t))$, então existe $k \in \mathbf{N}$ tal que $\lambda_{k} \in I_{3}(A)$. 
Prova- Se $\lambda \in I_{3}(A)$ temos que $(\lambda I-A)$ não é injetor e $R(\lambda I-A)=X$. Logo existe $x_{0} \in \mathcal{D}(A), x_{0} \neq 0$, tal que $(\lambda I-A) x_{0}=0$. Pelo Lema (1.1) temos que $\left(e^{\lambda t} I-T(t)\right) x_{0}=B_{\lambda}(t)(\lambda I-A) x_{0}=0$. Ou seja, $\left(e^{\lambda t} I-T(t)\right)$ não é injetor.

Para a segunda afirmativa temos que $\left(e^{\lambda t} I-T(t)\right)$ não é injetor e $R\left(e^{\lambda t} I-T(t)\right)=$ $X$. Logo, existe $x_{0} \neq 0$ tal que $\left(e^{\lambda t} I-T(t)\right) x_{0}=0$. Desse modo, a função $\varphi(s)=e^{-\lambda s} T(s) x_{0}$ é $t$-periódica e $\varphi(0)=x_{0}$. Logo, existe um coeficiente da expansão de $\varphi$ em série de Fourier não nulo. Seja $k \in \mathbf{N}$ tal que

$$
x_{k}=\frac{1}{t} \int_{0}^{t} e^{(-2 \pi i k / t) s} e^{-\lambda s} T(s) x_{0} d s \neq 0 .
$$

Com isso

$$
\frac{T(h)-I}{h} x_{k}=\frac{e^{\lambda_{k} h}-1}{h} x_{k} .
$$

E portanto, $x_{k} \in \mathcal{D}(A)$ e $A x_{k}=\lambda_{k} x_{k}$. Ou seja, $\lambda_{k} I-A$ não é injetor. Como $y \in X$ existe $x \in X$, tal que $\left(e^{\lambda t} I-T(t)\right) x=y$, concluímos que $\left(\lambda_{k} I-A\right) B_{\lambda_{k}}(t) x=y$. Isto é, $R\left(\lambda_{k} I-A\right)=X$.

Proposição 1.2 Sejam $T(t)$ um $C_{0}$-semigrupo e A seu gerador infenitesimal.

1. $S e \lambda \in I I_{3}(A)$, então $e^{\lambda t} \in I I_{3}(T(t)) \cup I I I_{3}(T(t))$.

2. Se $e^{\lambda t} \in I I_{3}(T(t))$, então existe $k \in \mathbf{N}$ tal que $\lambda_{k} \in I I_{3}(A) \cup I_{3}(A)$.

Prova- A não injetividade segue como a proposição anterior. Resta somente verificar que $R\left(e^{\lambda t} I-T(t)\right) \neq X$. Caso não ocorra, utilizando o lema acima podemos demonstrar que $R(\lambda I-A)=X$. O que é uma contradição.

Novamente, pela demonstração da Proposição (1.1) temos que existe $k \in \mathbf{N}$ tal que $\left(\lambda_{k} I-A\right)$ não é injetor. Com o auxílio do Lema (1.1) é imediato que $\overline{R\left(\lambda_{k} I-A\right)}=X$.

Proposição 1.3 Sejam $T(t)$ um $C_{0}$-semigrupo e $A$ seu gerador infinitesimal.

1. Se $\lambda \in I I I_{3}(A)$, entâo $e^{\lambda t} \in I I I_{3}(T(t))$.

2. Se $e^{\lambda t} \in I I I_{3}(T(t))$, então existe $k \in \mathbf{N}$ tal que $\lambda_{k} \in P \sigma(A)$. 
Prova- A não injetividade decorre como nos outros casos. Como $\overline{R(\lambda I-A)} \neq X$, segue, do Teorema de Hahn Banach [11], que existe $\xi \in X^{\prime}$, $\xi \neq 0$ tal que $\xi((\lambda I-A) x)=0$, para todo $x \in \mathcal{D}(A)$. Portanto,

$$
\xi\left(\left(e^{\lambda t} I-T(t)\right) x\right)=0 \text { para todo } x \in X .
$$

Logo, $R\left(e^{\lambda t} I-T(t)\right)$ não é denso em $X$.

Para a segunda afirmativa basta verificarmos a não injetividade de $\left(\lambda_{k} I-A\right)$ para algum $k \in \mathbf{N}$. O que é imediato a partir da Proposição (1.1).

Proposição 1.4 Sejam $T(t)$ um $C_{0}$-semigrupo e A seu gerador infinitesimal.

1. Se $\lambda \in I I I_{1}(A)$ e $\lambda_{n} \notin P \sigma(A)$, para todo $n \in \mathrm{N}$, então $e^{\lambda t} \in R \sigma(T(t))$.

2. Se $e^{\lambda t} \in I I I_{1}(T(t))$, então $\lambda_{n} \notin P \sigma(A)$, para todo $n \in \mathrm{N}$ e existe $k \in \mathrm{N}$ tal que $\lambda_{k} \in I I I_{1}(A)$.

Prova- A injetividade de $\left(\lambda_{k} I-A\right)$ para algum $k \in \mathrm{N}$ implica a injetividade de $\left(e^{\lambda t} I-T(t)\right)$. O fato de $R\left(e^{\lambda t} I-T(t)\right)$ não ser denso segue como o caso anterior.

Se para algum $n \in \mathbb{N},\left(\lambda_{n} I-A\right)$ não é injetor então $\left(e^{\lambda t} I-T(t)\right)$ também não é injetor. Ainda, para todo $n \in \mathrm{N},\left(\lambda_{n} I-A\right)^{-1}$ é limitado. Isto segue do fato que

$$
\left(\lambda_{n} I-A\right)^{-1} x=\left(e^{\lambda t} I-T(t)\right)^{-1} B_{\lambda_{n}}(t) x \text { para todo } x \in R\left(\lambda_{n} I-A\right) .
$$

Resta verificar que existe $k \in \mathrm{N}$, tal que $\overline{R\left(\lambda_{k} I-A\right)} \neq X$. Como $R\left(e^{\lambda t} I-T(t)\right)$ não é denso em $X$, segue que existe $\xi \in X^{\prime}, \xi \neq 0$, tal que $\xi\left(\left\{e^{\lambda t} I-T(t)\right) x\right)=0$, para todo $x \in X$. Para cada $x \in X$, consideremos a função $t$-periódica, $\varphi_{x}(s)=e^{-\lambda s} \xi(T(s) x)$. Conseqüentemente, existe $x_{0} \in X$ tal que $\xi\left(x_{0}\right) \neq 0$. Neste caso; existe $k \in \mathrm{N}$ tal que o k-ésimo coeficiente da série de Fourier de $\varphi_{x_{0}}$ é não nulo. Consideremos

$$
\xi_{k}(x)=\frac{1}{t} \int_{0}^{t} e^{-(\lambda+2 k \pi i / t) s} \xi(T(s) x) d s .
$$

Então $\xi_{k} \in X^{\prime}$ e $\xi_{k} \neq 0$. Tomando $x \in \mathcal{D}(A)$, temos $\xi_{k}\left(\left(\lambda_{k} I-A\right) x\right)=0$. Ou seja, $R\left(\lambda_{k} I-A\right)$ não é denso em $X$.

Proposição 1.5 Sejam $T(t)$ um $C_{0}$-semigrupo e A seu gerador infinitesimal.

1. Se $\lambda \in I I I_{2}(A)$ e $\lambda_{n} \notin P \sigma(A)$, para todo $n \in \mathrm{N}$, então $e^{\lambda t} \in I I I_{2}(T(t))$. 
2. Se $e^{\lambda t} \in I I I_{2}(T(t))$, então $\notin P \sigma(A)$, para todo $n \in \mathrm{N}$ e existe $k \in \mathrm{N}$ tal que $\lambda_{k} \in R \sigma(A)$.

Prova- A injetividade de $\left(e^{\lambda t} I-T(t)\right)$ é imediata. A não continuidade de $\left(e^{\lambda t} I-T(t)\right)^{-1}$ decorre da relação

$$
(\lambda I-A)^{-1} x=\left(e^{\lambda t} I-T(t)\right)^{-1} B_{\lambda}(t) x \text { para todo } x \in R(\lambda I-A) .
$$

Como $\overline{R(\lambda I-A)} \neq X$ temos que existe $\xi \in X^{\prime}$ tal que $\xi \neq 0$ e $\xi((\lambda I-A) x)=0$, para todo $x \in \mathcal{D}(A)$. E isso acarreta que $\xi\left(\left(e^{\lambda t} I-T(t)\right) x\right)=0$, para todo $x \in X$.

Tendo em vista as proposições anteriores é imediato a segunda parte deste resultado.

Proposição 1.6 Sejam $T(t)$ um $C_{0}$-semigrupo e A seu gerador infinitesimal.

1. Se $\lambda \in I_{2}(A)$ e $\lambda_{n} \notin P \sigma(A) \cup R \sigma(A)$, para todo $n \in \mathbf{N}$, então $e^{\lambda t} \in$ $C \sigma(T(t))$.

2. Se $e^{\lambda t} \in I_{2}(T(t))$, então $\lambda_{n} \notin P \sigma(A) \cup R \sigma(A)$ e $\lambda_{n} \in I_{2}(A) \cup I_{1}(A)$, para todo $n \in \mathbf{N}$.

Prova- A injetividade e a não continuidade decorre de argumentos análogos aos anteriores. Temos que $\overline{R\left(e^{\lambda t} I-T(t)\right)} \neq X$ não ocorre pois isso ocasionaria uma contradição com a hipótese assumida.

É fácil ver que para todo $n \in \mathbf{N}$ temos que $\left(\lambda_{n} I-A\right)$ é injetor. Da igualdade $\left(\lambda_{n} I-A\right) B_{\lambda_{n}}(t) x=\left(e^{\lambda t} I-T(t)\right) x$, para todo $x \in X$, temos que $R\left(\lambda_{n} I-A\right)=X$.

Proposição 1.7 Sejam $T(t)$ um $C_{0}$-semigrupo e $A$ seu gerador infinitesimal.

1. Se $\lambda \in I I_{2}(A)$ e $\lambda_{n} \notin P \sigma(A) \cup R \sigma(A)$, para todo $n \in \mathrm{N}$, então $e^{\lambda t} \in$ $I I_{2}(T(t))$.

2. $S e e^{\lambda t} \in I I_{2}(T(t))$, então $\lambda_{n} \notin P \sigma(A) \cup R \sigma(A)$ e $\lambda_{n} \in C \sigma(A) \cup \rho(A)$, para todo $n \in \mathbf{N}$.

Prova- $O$ resultado é imediato a partir dos resultados anteriores. 
Pr osição 1.8 Sejam $T\left(t i\right.$ un $C_{0}$-semigrupo e A seu gerador infinitesimal.

; Se $\lambda \in I_{1}(A)$ e $\lambda_{n} \notin P \sigma(A) \cup R \sigma(A)$, para todo $n \in \mathbf{N}$, entâo $e^{\lambda t} \in$ $C \sigma(T(t)) \cup \rho(T(t))$.

$S e e^{\lambda t} \in I_{1}(T(t))$, entâo $\lambda \in I_{1}(A)$

Sva- A existência de $(\lambda I-A)^{-1}$ nos garante que $\left(e^{\lambda t} I-T(t)\right)$ é inversivel.

Pelo Lema (1.1) temos que $(\lambda I-A)^{-1}$ existe, é limitado e $R(\lambda I-A)=X$.

Proposição 1.9 Sejam $T(t)$ um $C_{0}$-semigrupo e A seu gerador infinitesimal.

1. Se $\lambda \in I I_{1}(A)$ e $\lambda_{n} \notin P \sigma(A) \cup R \sigma(A)$, para todo $n \in \mathrm{N}$, então $e^{\lambda t} \in$ $C \sigma(T(t)) \cup \rho(T(t))$.

2. Se $e^{\lambda t} \in I_{1}(T(t))$, então $\lambda \in \rho(A)$.

Podemos resumir essas proposições nas seguintes inclusões espectrais em relação à divisão do espectro em pontual, residual e contínuo.

Teorema 1.7 Sejam $T(t)$ um $C_{0}$-semigrupo e A seu gerador infinitesimal.

1. Se $\lambda \in P \sigma(A)$, então $e^{\lambda t} \in P \sigma(T(t))$.

2. Se $e^{\lambda t} \in P \sigma(T(t))$, então existe $k \in \mathrm{N}$ tal que $\lambda_{k} \in P \sigma(A)$.

Teorema 1.8 Sejam $T(t)$ um $C_{0}$-semigrupo e A seu gerador infinitesimal.

1. Se $\lambda \in R \sigma(A)$ e para todo $n \in \mathrm{N}$ temos que $\lambda_{n} \notin P \sigma(A)$, então $e^{\lambda t} \in R \sigma(T(t))$.

2. Se $e^{\lambda t} \in R \sigma(T(t))$, entâo para todo $n \in \mathrm{N}$ temos que $\lambda_{n} \notin P \sigma(A)$ e existe $k \in \mathbf{N}$ tal que $\lambda_{k} \in R \sigma(A)$.

Teorema 1.9 Sejam $T(t)$ um $C_{0}$-semigrupo e $A$ seu gerador infinitesimal.

1. Se $\lambda \in C \sigma(A)$ e $\lambda_{n} \notin P \sigma(A) \cup R \sigma(A)$ para todo $n \in \mathbb{N}$, então $e^{\lambda t} \in$ $C \sigma(T(t))$. 


\section{Capítulo 2}

\section{Álgebra de Banach}

Neste capítulo introduzimos a noção de álgebra, isto é, um espaço vetorial com uma multiplicação definida entre vetores que, juntamente com a adição vetorial e a multiplicação por escalar possui determinadas propriedades. Na Seção (2.2) introduzimos o conceito de álgebra de Banach. Na Seção (2.3) apresentamos o conceito de espectro de elementos de uma álgebra de Banach. Nas seções (2.4) e (2.5) introduzimos a Teoria de Representação e a Topologia de Gelfand para álgebras de Banach. A primeira terá um papel importante na Seção (2.6) para mostrar que o Teorema Espectral é verdadeiro para semigrupos eventualmente contínuos e a segunda, através de uma forte relação com a topologia fraca*, nos permitirá definir uma topologia em um determinado conjunto de ideais e concluir resultados a respeito da compacidade deste conjunto.

\section{1 Álgebra}

Definição 2.1 Diremos que $\mathcal{U}$ é uma álgebra sobre o corpo $\Phi$ se seus elementos admitem três operações: adição, multiplicação e multiplicação por escalar, sujeitas às seguintes condições:

1. $\mathcal{U}$ com a adiçãa e a multiplicação por escalar é um espaço vetorial.

2. a multiplicação é uma operação fechada e associativa.

3. adição e multiplicação são distributivas: $x(y+z)=x y+x z$ para todo $x, y, z \in \mathcal{U}$. 
4. multiplicação e multiplicaşão por escala comutam: $\alpha x \beta y=\alpha \beta(x y)$ para todo $x, y \in \mathcal{U}$ e $\alpha, \beta \in \Phi$.

Outras condiçôes serão impostas algumas vєzes:

5. existe um elemento identidade $e$ tal que ex $=x \epsilon=x$, para todo $x \in \mathcal{U}$.

6. multiplicaçâo é comutativa: $x y=y x$ para todo $x, y \in \mathcal{U}$.

Diremos que $\mathcal{U}$ é uma álgebra com elemento identidade se 5 é verificada e $\mathcal{U}$ é uma álgebra comutativa se 6 for verdadeira.

Se $\mathcal{U}_{1}$ contido em $\mathcal{U}$ é uma álgebra, então $\mathcal{U}_{1}$ é chamado uma subálgebra de $\because$ ?.

D.sfinição 2.2 Seja $\mathcal{U}$ uma álgebra com elemento identidade e. Diremos que um eleinento $x \in \mathcal{U}$ é regular se existe um elemento $x^{-1} \in \mathcal{U}$, chamado o inverso de $x$, tal que $x x^{-1}=x^{-1} x=e$. Caso contrário, diremos que $x$ é singular. Se existe $y \in \mathcal{U}$ tal que $x y=e$ (resp. $y x=e)$, dizemos que $y$ é inverso à direita (resp. à esquerda) de $x$. Se todo elemento não nulo de $\mathcal{U}$ é regular, diremos que $\mathcal{U}$ é uma álgebra com divisão.

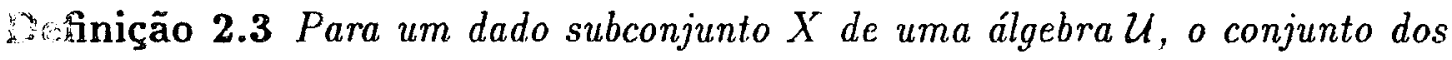
elementos de $\mathcal{U}$ que comutam com todo elemento de $X$ é chamado o comutante lo $X$ em $U$ e é denotado por $X^{c}$.

Notemos que $X^{c} \subset Y^{c}$, se $X \supset Y$ e que $X \subset X^{c c}$. Uma consequência é que $X^{c c c} \subset X^{c}$. Por outro lado, como todo elemento de $X^{c c}$, por definição, comuta com todo elemento de $X^{c}$, temos também $X^{c c c} \supset X^{c}$. Logo, $X^{c c c}=X^{c}$ e portanto $X^{c^{n+2}}=X^{c^{n}}, n \geq 1$.

Teorema 2.1 $O$ comutante de $X$ em $\mathcal{U}$ é uma subálgebra de $\mathcal{U}$. Se todos os elementos de $X$ comutam entre si então $X^{c c}$ é uma subálgebra comutativa de $\mathcal{U}$. Além disso, se $\mathcal{U}$ é uma álgebra com elemento identidade, então $X^{c}$ também o é. Se $x \in X^{c}$ é regular em $\mathcal{U}$, então $x^{-1} \in X^{c}$.

Prova- Se $x, y \in X^{c}$ e $z \in X$ então $x z=z x$ e $y z=z y$. Isto implica que $(x+y) z=x z+y z=z x+z y=z(x+y)$ e $(\alpha x) z=\alpha(z x)=z(\alpha x)$ e $x y z=x z y=$ $z x y$. Portanto, $x+y, \alpha x, x y$ estão todos em $X^{c}$ e isto é suficiente para mostrar 
que $X^{c}$ é uma álgebra.

Se todos os elementos de $X$ comutam entre si então $X \subset X^{c}$ e portanto $X^{c c} \subset X^{c}$. Como, por definição, todo elemento de $X^{r c c}$ comuta com todo elemento de $X^{c}$, segue que $X^{c c}$ é uma subálgebra comutativa. De fato,

se $x, y \in X^{c c}$, então $x z=z x$ e $y z=z y$ para todo $z \in X^{c}$. Como $X^{c c} \subset X^{c}$. temos que $x y=y x$.

Se $\mathcal{U}$ possui um elemento identidade $e$, então $e \in X^{c}$. Além disso, se $x \in X^{c}$ é regular e $z \in X$ então

$x^{-1} z=x^{-1} z\left(x x^{-1}\right)=x^{-1}(x z) x^{-1}=x^{-1} x z x^{-1}=z x^{-1}$. Ou seja, $x^{-1} \in X^{c}$.

\section{2 Álgebras de Banach}

Definição 2.4 Diremos que uma álgebra $\mathcal{U}$ é uma álgebra topológica se $\mathcal{U}$ é $u m$ espaço vetorial topológico e para todo $x, y \in \mathcal{U}$ e qualquer vizinhança $V(x y)$ de $x y$, existem vizinhanças $V(x)$ de $x$ e $V(y)$ de $y$ tais que $x V(y) \subset V(x y)$ e $V(x) y \subset V(x y)$.

O efeito desta definição é requerer que $x y$ seja contínua em $x$ e $y$ separadamente.

Teorema 2.2 Seja $X$ um subconjunto de uma álgebra topológica $\mathcal{U}$. Então o comutante, $X^{\mathrm{c}}$, de $X$ em $\mathcal{U}$ é um conjunto fechado.

Prova- Suponha que $X^{c}$ não seja fechado. Então existe $y \in \overline{X^{c}}-X^{c}$ e existe $z \in X$ tal que $y z-z y \neq 0$. Então encontramos um vizinhança $V(y z-z y)$ de $y z-z y$ que não contém o zero. Mas

$$
V(y z)-V(z y) \subset V(y z-z y), V(y) z \subset V(y z) \text { e } z V(y) \subset V(y z),
$$

logo,

$$
V(y) z-z V(y) \subset V(y z-z y) .
$$

Como $y \in \overline{X^{c}}$, existe $x \in V(y) \cap X^{c}$ e portanto $0=x z-z x \in V(y z-z y)$ o que é uma contradição.

Definição 2.5 Diremos que $\mathcal{B}$ é uma álgebra de Banach se é uma álgebra, que é um espaço de Banach $e$

$$
\|x y\| \leq\|x\|\|y\| .
$$

Quando o corpo $\Phi$ é real (resp. complexo) diremos que $\mathcal{B}$ é uma álgebra real (resp. complexa) de Banach. 
A desigualdade

$$
\left\|x_{1} y_{1}-x_{2} y_{2}\right\| \leq\left\|x_{1}\right\|\left\|y_{1}-y_{2}\right\|+\left\|y_{2}\right\|\left\|x_{1}-x_{2}\right\|
$$

nos mostra que $x y$ é uma função contínua em ambas as variáveis. Em particular, $\mathcal{B}$ é uma álgebra topológica.

Uma álgebra $\mathcal{U}$ satisfazendo todas as condições para uma álgebra de Banach, exceto o completamento, é chamada um álgebra normada; uma tal álgebra pode ser imersa em uma álgebra de Banach $\mathcal{B}$.

Se uma álgebra de Banach tem elemento identidade $e$ (distinto de 0), então necessariamente $\|e\| \geq 1$. Em geral, podemos assumir que $\|e\|=1$, pois $\mathcal{B}$ é sempre isomorfa a uma álgebra $\mathcal{C}$ com elemento identidade de norma 1.

No decorrer deste capítulo o símbolo $\mathcal{B}$ denotará sempre uma álgebra complexa de Ba ach com elemento identidade $e$. A multiplicação não é comutativa, a menos que o contrário seja especificado. Todas afirmações a respeito de limites e convergência são entendidas em termos da norma do espaço $\mathcal{B}$.

Teorema 2.3 Todo elemento na bola aberta $\|e-x\|<1$ é regular $e$

$$
x^{-1}=e+\sum_{n=1}^{\infty}(e-x)^{n} \text {. }
$$

Prova- A série acima converge absolutamente em virtude da hipótese $\|e-x\|<1$. Logo a série acima define um elemento em $\mathcal{B}$. É fácil ver que

$$
\left(e+\sum_{n=1}^{\infty}(e-x)^{n}\right) x=x\left(e+\sum_{n=1}^{\infty}(e-x)^{n}\right)=e .
$$

Corolário 2.1 Sejam $x \in \mathcal{B}$ e $\lambda \in$ C. Se $|\lambda|>\|x\|$, então $\lambda e-x$ é regular $e$

$$
(\lambda e-x)^{-1}=\sum_{n=1}^{\infty} \lambda^{-n} x^{n-1} \text {. }
$$

Teorema 2.4 Os elementos regulares formam um conjunto aberto em $\mathcal{B}$.

Prova- Denotemos o conjunto dos pontos regulares por $\mathcal{O}$. Para $x_{0} \in \mathcal{O}$ a bola aberta $\left\|x-x_{0}\right\|<\left\|x_{0}^{-1}\right\|^{-1}$ está contida em $\mathcal{O}$.

Como consequêencia temos

Teorema 2.5 A função $x \rightarrow x^{-1}$ é contínua em $\mathcal{O}$. 


\subsection{Teoria Espectral para Álgebras de Banach}

Consideremos o inverso de $\lambda e-a$ como uma função de $\lambda$ para um $a \in \mathcal{B}$ fixo. Para $a \in \mathcal{B}$ definiremos o conjunto resolvente de $a$ e o espectro de $a$. Como foi visto no Capítulo 1, para operadores lineares temos a divisão do espectro em espectro pontual, contínuo e residual, porém para uma álgebra arbitrária essa divisão não faz sentido, assim estaremos interessados no conjunto resolvente e seu complemento, o espectro.

Definição 2.6 Diremos que $\lambda$ pertence ao conjunto resolvente $\rho(a)$, se $\lambda e-a$ é regular. Caso contrário, diremos que $\lambda$ pertence ao espectro $\sigma(a)$. Para $\lambda \in \rho(a)$, o inverso de $(\lambda e-a)$ será denotado por $R(\lambda, a)$ (algumas vezes também será representado por $\left.(\lambda e-a)^{-1}\right)$ e chamado operador resolvente de a.

Para o caso particular em que $\mathcal{B}=L(X)$, com $X$ um espaço de Banach, não há ambigüidade quando consideramos o espectro de um operador $A \in L(X)$ usando a definição acima ou a definição dada no Capítulo 1. Para ver isso, basta mostrarmos que o complemento destes dois conjuntos são iguais. De fato, se $\lambda$ é ponto regular de $A$ então $(\lambda I-A)^{-1} \in L(X)$. Logo, $\lambda \in \rho(A)$. Reciprocamente, se $\lambda \in \rho(A)$ é fácil ver que $R(\lambda I-A)=X$.

Teorema 2.6 O resolvente é um conjunto aberto. Além disso, $R(\lambda, a)$ é uma função analítica de $\lambda$.

Prova- Seja $\lambda_{0} \in \rho(a)$. Consideremos a seguinte vizinhança de $\lambda_{0}$ :

$$
\left|\lambda-\lambda_{0}\right|<\left\|R\left(\lambda_{0}, a\right)\right\|^{-1} \text {. }
$$

A série

$$
\sum_{n=1}^{\infty}\left(\left(\lambda-\lambda_{0}\right) R\left(\lambda_{0}, a\right)\right)^{n}
$$

converge absolutamente na vizinhança dada. Tomando

$$
y=R\left(\lambda_{0}, a\right)\left(e+\sum_{n=1}^{\infty}\left(\left(\lambda_{0}-\lambda\right) R\left(\lambda_{0}, a\right)\right)^{n}\right),
$$

temos que $y(\lambda e-a)=(\lambda e-a) y=e$. 
Definição 2.7 Dado $a \in \mathcal{B}$, definimos o raio espectral $r(a)$, por

$$
r(a)=\sup \{|\lambda|: \lambda \in \sigma(a)\} .
$$

$\mathrm{O}$ resultado a seguir tem dupla importância. Primeiro pelo fato de determinar um modo alternativo para o cálculo do raio espectral de um elemento $a$, segundo por representar um primeiro Teorema Espectral.

Teorema 2.7 Seja $\mathcal{B}$ uma álgebra complexa normada com identidade. Sejam $a \in \mathcal{B}$ e $p$ um polinômio com coeficientes complexos. Então

$$
\sigma(p(a))=p(\sigma(a))
$$

Prova- É imediato ò resultado para polinômios constantes. Também é fácil ver que $\sigma(\alpha z)=\alpha \sigma(z)$ para todo $z \in \mathcal{B}$ e $\alpha \in \mathrm{C}$. Seja $p$ um polinômio de grau $n \geq 1$. Basta provarmos o resultado para polinômio com coeficiente dominante unitário. Consideremos $p(t)-\lambda, \lambda \in \mathbf{C}$. Como $p(t)-\lambda$ pode ser fatorado completamente em termos lineares,

$$
p(t)-\lambda=\left(t-\beta_{1}\right)\left(t-\beta_{2}\right) \ldots\left(t-\beta_{n}\right),
$$

temos que

$$
p(a)-\lambda=\left(a-\beta_{1}\right)\left(a-\beta_{2}\right) \ldots\left(a-\beta_{n}\right) .
$$

Se $\lambda \in \sigma(p(a))$ então para algum $j, 1 \leq j \leq n, a-\beta_{j}$ e é singular, ou seja, $\beta_{j} \in \sigma(a)$. Portanto, $p\left(\beta_{j}\right) \in p(\sigma(a))=\{p(\lambda): \lambda \in \sigma(a)\}$. Lembrando que $p\left(\beta_{j}\right)=\lambda$ temos a primeira inclusão. A outra segue de modo análogo.

Corolário 2.2 Sejam $X$ um espaço vetorial complexo de Banach e $L(X)$ a álgebra dos operadores lineares contínuos. Se $A \in L(X)$, então para qualquer polinômio $p$ com coeficientes complexos temos

$$
\sigma(p(A))=p(\sigma(A))
$$

Teorema 2.8 O raio espectral verifica às seguintes propriedades:

1. $r\left(a^{k}\right)=(r(a))^{k}$.

2. $r(\alpha a)=|\alpha| r(a)$. 
3. $r(a) \leq\|a\|$.

4. $r(a) \leq \varliminf_{n \rightarrow \infty}\left\|a^{n}\right\|^{\frac{1}{n}}$.

Prova- Do teorema anterior segue imediatamente as duas primeiras afirmativas. A terceira é uma consequência do Corolário (2.1). Portanto,

$$
r(a)^{n} \leq\left\|a^{n}\right\| .
$$

Ou ainda,

$$
r(a) \leq\left\|a^{n}\right\|^{\frac{1}{n}} .
$$

Tomando o limite inferior temos

$$
r(a) \leq \lim _{n \rightarrow \infty}\left\|a^{n}\right\|^{\frac{1}{n}} .
$$

O próximo resultado nos mostra que podemos melhorar a desigualdade 4 do Teorema (2.8). Na verdade será provado que

$$
r(a)=\lim _{n \rightarrow \infty}\left\|a^{n}\right\|^{\frac{1}{n}} .
$$

Teorema 2.9 Sejam $\mathcal{B}$ uma álgebra complexa de Banach com identidade e a um elemento de $\mathcal{B}$.

1. Para $|\lambda|>r(a), R(\lambda, a)$ existe e é dado por

$$
\sum_{n=1}^{\infty} \lambda^{-n} a^{n-1}
$$

2. Se a série (2.1) converge para $|\lambda|=r(a)$, então $R(\lambda, a)$ existe e $R(\lambda, a)=$ $\sum_{n=1}^{\infty} \lambda^{-n} a^{n-1}$.

3. A série (2.1) diverge para $|\lambda|<r(a)$.

Prova- Primeiramente observemos, que se $\lambda$ é tal que $|\lambda|>r(a)$, então $\lambda \in \rho(a)$. Assim, pelo Teorema (2.6), a função $R(\lambda, a)$ é analítica para todo ponto fora do círculo fechado de raio $r(a)$, logo pode ser expandida em série de Taylor em torno do ponto $\infty$. Por outro lado, pelo Corolário $(2.1), R(\lambda, a)$ é dado pela série (2.1) para $|\lambda|>\|a\|$. Portanto concluímos que a expansão em série de Taylor de 
$R(\lambda, a)$ é igual a série (2.1).

Para a segunda parte, basta vermos que a convergência da série (2.1) nos permite concluir que seu limite é o inverso de $\lambda e-a$.

Para a última afirmativa, notemos que se existe $\lambda_{0},\left|\lambda_{0}\right|<r(a)$, tal que a série (2.1) converge, então para todo $\lambda$ fora do círculo de raio $\left|\lambda_{0}\right|,(\lambda e-a)$ é regular. O que é uma contradição.

Por (1) e (3) do teorema acima temos que o raio de convergência da série (2.1) é $r(a)$. Por outro lado podemos calcular o raio de convergência de qualquer série pelo seguinte limite

$$
\varlimsup_{n \rightarrow \infty}\left\|a^{n-1}\right\|^{\frac{1}{n}} .
$$

Mas

$$
\varlimsup_{n \rightarrow \infty}\left\|a^{n}\right\|^{\frac{1}{n}} \leq \varlimsup_{n \rightarrow \infty}\left\|a^{n-1}\right\|^{\frac{1}{n}} \varlimsup_{\lim _{n \rightarrow \infty}}\|a\|^{\frac{1}{n}}=r(a) .
$$

Juntamente com a parte 4 do Teorema (2.8), obtemos

$$
\lim _{n \rightarrow \infty}\left\|a^{n}\right\|^{\frac{1}{n}}=r(a) .
$$

Em particular, se $A \in L(X) \operatorname{com} X$ espaço de Banach complexo, temos que

$$
\lim _{n \rightarrow \infty}\left\|A^{n}\right\|^{\frac{1}{n}}=r(A) .
$$

Teorema 2.10 O espectro de a é um conjunto não vazio e compacto.

Prova- Mostremos somente a primeira afirmação, pois a outra é imediata a partir das conclusões anteriores. Se $\sigma(a)$ é vazio, então $R(\lambda, a)$ é uma função inteira e limitada e pelo Teorema de Liouville, $R(\lambda, a)$ é ser constante. Além disso, $R(\lambda, a) \equiv 0$. O que é uma contradição.

Corolário 2.3 Seja $\mathcal{U}$ uma álgebra complexa normada com elemento identidade. Então o espectro de cada a $\in \mathcal{U}$ é não vazio.

\subsection{Teoria de Representação de Gelfand}

O resultado mais importante desta seção é o Teorema (2.18) pois nos permite obter, entre outras informações, uma nova caracterização do espectro de um elemento de uma álgebra de Banach. Esse resultado será aplicado para uma particular subálgebra a ser definida na Seção (2.6). Assim, iniciamos com definições 
e resultados gerais sobre teoria de ideias e a partir do Teorema de Gelfand e do Teorema (2.17) conseguimos obter o Teorema de Representação de Gelfand. Finalizando a seção, mostramos que é possivel determinar uma correspondência um a um entre os ideais maximais $\mathcal{B}$ e os funcionais lineares multiplicativos definidos sobre $\mathcal{B}$. Essa correspondência é importante na caracterização da Topologia de Gelfand que será definida na Seção (2.5).

Definição 2.8 Seja $\mathcal{B}$ uma álgebra complexa de Banach comutativa com elemento identidade.

1. Um subconjunto $I$ de $\mathcal{B}$ é um ideal de $\mathcal{B}$ se $x-y \in I$ e $x z \in I$ para todo $x, y \in I$ e $z \in \mathcal{B}$.

2. Um ideal I é próprio se I é diferente de $\{0\}$ e $\mathcal{B}$.

3. Um ideal $M$ é maximal se é diferente de toda álgebra e não está contido propriamente em qualquer outro ideal.

É imediato que

Teorema 2.11 O fecho de um ideal próprio é um ideal próprio.

Corolário 2.4 Um ideal maximal é necessariamente fechado.

Teorema 2.12 Todo ideal próprio está contido em um ideal maximal.

Prova- Dado $I$ um ideal próprio de $\mathcal{B}$, basta considerarmos a classe de todos os ideais próprios contendo $I$ e aplicar o Lema de Zorn.

Teorema 2.13 Um elemento $x \in \mathcal{B}$ é regular se, e somente se $x \notin M$, para todo ideal maximal $M$ de $\mathcal{B}$.

Prova- Se $x$ é regular e $x \in M$, então $e \in M$, o que implica que $M=\mathcal{B}$. Reciprocamente, se $x$ é singular, tomando $I=\mathcal{B} x$ temos que $I$ é um ideal próprio contendo $x$. Logo, pelo Teorema (2.12) $x$ está em algum ideal maximal $M$. 
Definição $2.9 O$ radical $\varrho$, de un : álgebra comutativa com elemento identidade $\mathcal{B}$, é a interseção de todos os ide ss maximais de $\mathcal{B}$. Se $\varrho$ consiste apenas do elemento zero, dizemos que $\mathcal{B}$ é umia álgebra semi-simples.

Seja $I$ um ideal de uma álgebra complexa comutat: $: \mathcal{U}$ com elemento identidade. Por definição, $I$ é um subespaço de $\mathcal{U}$ e então po emos definir uma relação de congruência modulo $I$. Temos assim o espaço quociente $\mathcal{U} / I$. Além disso, $\mathcal{U} / I$ tem uma estrutura de álgebra, chamada álgebra quociente. $O$ elemento zero de $\mathcal{U} / I$ é $I$, e o elemento identidade $E$ de $U / I$ é a classe determinada pelo elemento identidade de $\mathcal{U}$. A aplicação que leva $x \in \mathcal{U}$ na classe $X$ contendo $x$ é um homomorfismo de $\mathcal{U}$ sobre $\mathcal{U} / I$. Resumindo temos,

Teorema 2.14 Se $\mathcal{U}$ é uma álgebra complexa comutativa com elemento identidade e I um ideal de $\mathcal{U}$ então $\mathcal{U} / I$ é uma álgebra complexa comutativa com elemento identidade e existe um homomorfismo sobrejetor de $\mathcal{U}$ em $\mathcal{U} / I$. Além disso, se $\mathcal{B}$ é uma álgebra complexa de Banach comutativa com elemento identidade e I. um ideal fechado de $\mathcal{B}$ então $\mathcal{B} / I$ é uma álgebra complexa de Banach comutativa com elemento identidade com a norma

$$
\|X\|=\inf \{\|x\|: x \in X\} .
$$

Teorema 2.15 (Gelfand) Toda álgebra complexa de Banach com identidade $e$ com divisão é isomrfa ao corpo dos números complexos.

Prova- Dado $x \in \mathcal{B}$ existe $\lambda \in \mathrm{C}$ tal que $x-\lambda e$ é singular. Sendo $\mathcal{B}$ uma álgebra com divisão temos que $x-\lambda e=0$, ou seja, todo elemento de $\mathcal{B}$ pode ser escrito como um múltiplo escalar da identidade. É fácil ver que este escalar é único. Desse modo, podemos construir a seguinte aplicação que é um isomorfismo de álgebras:

$$
f: x \in \mathcal{B} \rightarrow \lambda \in \mathbf{C} .
$$

Para o caso em que $\mathcal{B}$ é real, o Teorema de Frobenius, I. N. Herstein, Topics in Algebra, garante que $\mathcal{B}$ é isomorfa ou aos números reais ou aos números complexos ou ao corpo dos quatérnios.

Teorema 2.16 Seja $\mathcal{B}$ uma álgebra complexa de Banach com identidade. Se $\mathcal{B}$ não contém ideais próprios então $\mathcal{B}$ é isomorfo ao corpo dos números complexos. 
Prova- Nas condições dadas temos que todo elemento de $\mathcal{B}$ é regular. Em outras palavras, $\mathcal{B}$ é uma álgebra com divisão. $O$ que mostra o teorema.

Teorema 2.17 Se $M$ é um ideal maximal contido numa álgebra complexa de Banach comutativa com elemento identidade, então $\mathcal{B} / M$ é isomorfo ao corpo dos números complexos. Em particular, existe um homomorfismo sobrejetor de $\mathcal{B}$ em $\mathbf{C}$ que associa a cada $x \in \mathcal{B}$ o número complexo $x(M)$ tal que

$$
x \equiv x(M) e(\bmod M) .
$$

Este homomorfismo satisfaz às propriedades:

1. $\left(x_{1}+x_{2}\right)(M)=x_{1}(M)+x_{2}(M)$.

2. $(\alpha x)(M)=\alpha x(M)$.

3. $\left(x_{1} x_{2}\right)(M)=x_{1}(M) x_{2}(M)$.

4. $e(M)=1$.

5. $x(M)=0$ se, e somente se $\in M$.

6. $|x(M)| \leq\|x\|$.

Prova- Pelo fato de $M$ ser um ideal maximal temos que $\mathcal{B} / M$ é corpo e portanto pelo Teorema (2.15) $\mathcal{B} / M$ é isomorfo ao corpo dos números complexos. Este isomorfismo leva $x \in \mathcal{B}$ em $x(M) \in \mathbf{C}$, onde $x(M) E$ é a imagem de $x$ pelo homomorfismo sobrejetor $\mathcal{B}$ em $\mathcal{B} / M$. Portanto, $x(M) e \in X$, isto é,

$$
x \equiv x(M) e(\bmod M)
$$

As cinco primeiras propriedades decorrem imediatamente do homomorfismo sobrejetor $\mathcal{B}$ em $\mathcal{B} / M$. A última afirmativa segue da desigualdade

$$
\|X\|=\|x(M) E\|=|x(M)|\|E\| \geq|x(M)| \text {. }
$$

Sejam $\mathcal{B}$ uma álgebra complexa de Banach comutativa com elemento identidade e $\mathcal{M}$ o conjunto de todos os ideais maximais de $\mathcal{B}$. Para cada $x \in \mathcal{B}$, definimos uma função de $\mathcal{M}$ em $\mathrm{C}$ por $\widehat{x}(M)=x(M)$, para todo $M \in \mathcal{M}$. Obtemos então o Teorema de Representação de Gelfand. 
Teorema 2.18 Seja $\mathcal{B}$ uma álgebra complexa de Banach comutativa com elemento identidade e. Seja $\mathcal{M}$ o conjunto de todos os ideais maximais em $\mathcal{B}$. As funções $\widehat{x}, x \in \mathcal{B}$, satisfazem às seguintes propriedades:

1. $\left(x_{1} \widehat{+} x_{2}\right)(M)=\widehat{x_{1}}(M)+\widehat{x_{2}}(M)$.

2. $(\widehat{\alpha x})(M)=\alpha \widehat{x}(M)$.

3. $\left(\widehat{x_{1} x_{2}}\right)(M)=\widehat{x_{1}}(M) \widehat{x_{2}}(M)$.

4. $\hat{e}(M)=1$.

5. $\widehat{x}(M)=0$ se, e somente se $x \in M$.

6. $\sigma(x)=\widehat{x}(\mathcal{M})$

7. o raio espectral $r(x)=\sup \{|x(M)|: M \in \mathcal{M}\} \leq\|x\|$.

8. um elemento $x$ é regular se, e somente se $x(M) \neq 0$, para todo $M \in \mathcal{M} e$ $x^{-1}(M)=x(M)^{-1}$.

9. se $M_{1} \neq M_{2}$, então existe $x \in \mathcal{B}$ tal que $\widehat{x}\left(M_{1}\right) \neq \widehat{x}\left(M_{2}\right)$.

Prova- As cinco primeiras propriedades decorrem imediatamente do Teorema (2.17). Um elemento $x \in \mathcal{B}$ é regular se, e somente se $x \notin M$, para todo $M \in \mathcal{M}$ e portanto, de acordo com (5) se, e somente se $x(M) \neq 0$, para todo $M \in \mathcal{M}$. Com isso podemos concluir que $\lambda \in \rho(x)$ se, e somente se $\lambda \neq x(M)$, para todo $M \in \mathcal{M}$. E disto decorre (6). A propriedade (7) segue imediatamente da definição de raio espectral e de (6). Das propriedades (4) e (6) podemos concluir facilmente (8). Finalmente, se $M_{1} \neq M_{2}$ existe pelo menos um elemento $x \in M_{1}$ tal que $x \notin M_{2}$ pois $M_{1}, M_{2} \in \mathcal{M}$. Por (5) o resultado segue.

Corolário 2.5 A aplicação que leva cada $x \in \mathcal{B}$ na função $\widehat{x}$ de $\mathcal{M}$ em $\mathrm{C} e ́$ um homomorfismo. Esta aplicação é um isomorfismo se, e somente se $\mathcal{B}$ é uma álgebra semi-simples.

Prova- Para a segunda afirmativa basta observarmos que o núcleo do homomorfismo é justamente o radical de $\mathcal{B}$. 
Definição 2.10 Diremos que uma função $\mu$ de $\mathcal{B}$ em $\mathrm{C}$, não identicamente nula, é $u m$ funcional linear multiplicativo sobre $\mathcal{B}$ se

1. $\mu(\alpha x+\beta y)=\alpha \mu(x)+\beta \mu(y)$.

2. $\mu(x y)=\mu(x) \mu(y)$.

para todo $x, y \in \mathcal{B}$ e $\alpha, \beta \in \mathbf{C}$.

Dada uma álgebra complexa de Banach comutativa com elemento identidade $\mathcal{B}$, do Teorema (2.17) temos que para cada ideal maximal $M$, existe um funcional linear multiplicativo $\mu$ sobre $\mathcal{B}$ definido por $\mu(x)=x(M)$. O teorema a seguir nos mostra que existe uma correpondência um a um entre os ideais maximais e os funcionais lineares multiplicativos sobre $\mathcal{B}$.

Teorema 2.19 Seja $\mathcal{B}$ uma álgebra complexa de Banach comutativa com elemento identidade. Se $\mu(x)$ é um funcional linear multiplicativo sobre $\mathcal{B}$ então $M=\{x: \mu(x)=0\}$ é um ideal maximal de $\mathcal{B}$ e $\mu(x)=x(M)$, para todo $x \in \mathcal{B}$.

Prova- É imediato ver que $M$ é um jdeal. Se $M$ não é maximal temos que existe um ideal próprio $I$ tal que $M \subset I$ e $M \neq I$, ou seja, existe $x_{0} \in I-M$, o que implica que $\mu\left(x_{0}\right) \neq 0$. Logo $\mu\left(x_{0}-\mu\left(x_{0}\right) e\right)=0$, ou seja, $x_{0}-\mu\left(x_{0}\right) e \in M$ e assim, $\mu\left(x_{0}\right) e \in I$. O que contradiz o fato de $I$ ser próprio. Portanto $M$ é maximal e temos o homomorfismo de álgebras

$$
x \in \mathcal{B} \rightarrow x(\bar{M}) \in \mathrm{C},
$$

onde $x-x(M) e \in M$. Deste modo concluímos que $\mu(x)=x(M)$.

Corolário 2.6 Se $\mu(x)$ é um funcional linear multiplicativo sobre $\mathcal{B}$, então $\mu$ é limitado $e\|\mu\| \leq 1$.

Prova- É imediata a partir da propriedade 6 do Teorema (2.17).

\subsection{Topologia de Gelfand}

Consideremos $\mathcal{B}$ uma álgebra complexa de Banach comutativa com elemento identidade, $\mathcal{M}$ o conjunto de todos os ideais maximais de $\mathcal{B}$ e para cada $x \in \mathcal{B}$ a função $\widehat{x}$ de $\mathcal{M}$ em $\mathbf{C}$ definida por $\widehat{x}(M)=x(M)$, para todo $M \in \mathcal{M}$. 
Nosso objetivo é definir um topologia em $\mathcal{M}$ que torna estas funções contínuas. Tomemos a topologia fraca determinada pela família $\{\widehat{x}\}_{x \in \mathcal{B}}$, isto é, a topologia menos fina com relação a qual todas $\widehat{x}$ são contínuas. Com isso os conjuntos abaixo formam uma base para $\mathcal{M}$.

$$
V\left(M_{0}, \widehat{x_{1}}, \ldots, \widehat{x_{n}}\right)=\left\{M \in \mathcal{M}:\left|x_{i}(M)-x_{i}\left(M_{0}\right)\right|<\epsilon, i=1, \ldots, n\right\} .
$$

Esta topologia é chamada a Topologia de Gelfand. Notemos que com esta topologia $\mathcal{M}$ é um espaço de Hausdorff. Mais ainda, do Teorema (2.19) e do Corolário (2.6) obtemos que os funcionais lineares multiplicativos formam um subconjunto do disco unitário $D$ de $\mathcal{B}^{\prime}$ onde $\mathcal{B}^{\prime}$ é o conjunto de todos os funcionais lineares limitados de $\mathcal{B}$. Logo, $\mathcal{M}$ pode ser visto como um subconjunto de $D$ e temos que a Topologia de Gelfand para $\mathcal{M}$ coincide com a topologia induzida pela topologia fraca* de $\mathcal{B}^{\prime}$.

Teorema 2.20 $O$ espaço $\mathcal{M}$ é compacto com relação à topologia de Gelfand.

Prova- Pelo Teorema de Banach-Alaoglu [11, pag. 228] temos que o disco unitário $D$, de $\mathcal{B}^{\prime}$ é compacto na topologia fraca*. Logo, basta demonstrar que $\mathcal{M}$ é um subespaço fechado de $D$. Seja $f \in \overline{\mathcal{M}} \cap D$. Então $f$ é um funcional linear limitado com $\|f\| \leq 1$. Como $f \in \overline{\mathcal{M}}$ temos que

$$
V(f, x, y, x y, e, \epsilon) \cap \mathcal{M} \neq \emptyset
$$

onde $\ddot{\epsilon}>0$ e $x, y$ são elementos arbitrários de $\mathcal{B}$. Portanto, existe um funcional linear multiplicativo $\mu$ tal que

$$
\begin{cases}\mu(x)-f(x) \mid<\epsilon, & |\mu(y)-f(y)|<\epsilon \\ |\mu(x y)-f(x y)|<\epsilon \mathrm{e} & |\mu(e)-f(e)|<\epsilon .\end{cases}
$$

Usando que $\mu(x y)=\mu(x) \mu(y)$ e $\mu(e)=1$ obtemos

$$
|f(x y)-f(x) f(y)|<(1+\|x\|+\|y\|) \epsilon
$$

e

$$
|f(e)-1|<\epsilon .
$$

para todo $\epsilon>0$. Ou seja, $f \in \mathcal{M}$, como querímos. 


\subsection{Teoria Espectral para Semigrupos Even- tualmente Contínuos}

Como já foi mencionado no Capítulo 1 e poderá ser verificado através dos exemplos do Capítulo 3, a inclusão inversa do Teorema (1.6) não é verdadeira em geral, isto é, nem todos os pontos do espectro de um semigrupo são provenientes, via aplicação exponencial, de pontos do espectro de seu gerador infinitesimal. O propósito desta seção é mostrar que, para uma dada classe de $C_{0^{-}}$ semigrupos, definidos em um espaço de Banach, podemos ter a inclusão contrária. Começaremos reunindo os fatos sobre álgebra de Banach suficientes para a conclusão do resultado.

Sejam $X$ um espaço de Banach, $T(t), t>0$ um $C_{0}$-semigrupo de operadores limitados lineares sobre $X$ e $A$ seu gerador infinitesimal.

Nosso objetivo é obtermos uma subálgebra de $L(X)$. Para isto, consideremos dois conjuntos

$$
\mathcal{T}=\{T(t): t>0\}
$$

e

$$
\mathcal{R}=\{R(\lambda, A): \lambda \in \rho(A)\} .
$$

Teorema 2.21 Para os conjuntos $\mathcal{T}$ e $\mathcal{R}$ temos que $\mathcal{T}^{c}=\mathcal{R}^{c}$ e $\mathcal{T}^{c c}=\mathcal{R}^{c c}$.

Prova- Basta provar a primeira afirmativa. Seja $B \in \mathcal{T}^{c}$. Pelo Corolário (1.1) segue que existe $\omega \in \mathbf{R}$ tal que se $\Re(\lambda)>\omega$ então $\lambda \in \rho(A)$ e para todo $x \in X$ temos,

$$
R(\lambda, A) x=\int_{0}^{\infty} e^{-\lambda s} T(s) x d s .
$$

Como $B$ é um operador linear limitado, temos que $B R(\lambda, A) x=R(\lambda, A) B x$ para todo $x \in X$. Além disso, para $x \in D(A), A B x=B A x$, o que implica $B R(\lambda, A) x=R(\lambda, A) B$, para todo $\lambda \in \rho(A)$. Logo, $B \in \mathcal{R}^{c}$.

Reciprocamente, sejam $B \in \mathcal{R}^{c}$ e $x \in \mathcal{D}\left(A^{2}\right)$. Então $B\left(D\left(A^{2}\right)\right) \subset D\left(A^{2}\right)$. Esta inclusâao segue da igualdade $D\left(A^{2}\right)=R\left(R(\lambda, A)^{2}\right)$.

Tomando $\gamma>\max \{0, \omega\}$ temos

$$
T(t) x=\lim _{n \rightarrow \infty} \frac{1}{2 \pi i} \int_{\gamma-i n}^{\gamma+i n} e^{\lambda t} R(\lambda, A) x d \lambda .
$$

Desde que $\mathcal{D}\left(A^{2}\right)$ é denso em $X$, segue que $B \in \mathcal{T}^{c}$. 
Teorema 2.22 A subálgebra $\mathcal{B}=\mathcal{T}^{c c}=\mathcal{R}^{c c}$ é uma subálgebra fechada comutativa de $L(X)$, com identidade $I$ e o espectro de qualquer operador $B \in \mathcal{B}$, relativo $\dot{a} \mathcal{B}$, é $\sigma(B)$. Além disso, $\mathcal{B} \supset \mathcal{T} \cup \mathcal{R}$.

O próximo teorema reúne alguns resultados a respeito da teoria de representação de Gelfand para a particular álgebra $\mathcal{B}$.

Teorema 2.23 Seja $\mathcal{M}$ o conjunto de todos os ideais maximais de $\mathcal{B}$ munido da topologia de Gelfand. Então $\mathcal{M}$ é um espaço de Hausdorff. A aplicação que leva $B \in \mathcal{B}$ na função $\widehat{B}$ de $\mathcal{M}$ em $\mathbf{C}$ é um homomorfismo contínuo. O espectro de $B$, relativo à $\mathcal{B}$, é precisamente $B(\mathcal{M})=\{B(M): M \in \mathcal{M}\}$. Além disso, $\sigma(B)=B(\mathcal{M})$.

Lema 2.1 Sejam $A$ um operador não limitado, densamente definido sobre $X e$ $z \notin \sigma(A)$. Então

$$
\sigma(R(z, A))=\{0\} \cup\left\{(z-\lambda)^{-1}: \lambda \in \sigma(A)\right\} .
$$

Prova- Pelo fato de $A$ não ser limitado e $R(R(z, A))=\mathcal{D}(A)$ temos que $0 \notin \rho(R(z, A))$. Se $\lambda \notin \sigma(A)$ o operador $S$ definido por

$$
S=(z-\lambda)(z I-A) R(\lambda, A)
$$

possui as seguintes propriedades:

1. $S$ comuta com $R(z, A)$.

2. $S$ é limitado.

3. $S\left((z-\lambda)^{-1} I-R(z, A)\right)=I$.

4. $\left((z-\lambda)^{-1} I-R(z, A)\right) S x=x$, para todo $x \in X$.

Portanto, $(z-\lambda)^{-1} \notin \sigma(R(z, A))$.

Reciprocamente, se $(z-\lambda)^{-1} \notin \sigma(R(z, A))$, considerando

$$
S=(I-(z-\lambda) R(z, A))^{-1} R(z, A),
$$

temos que $(\lambda I-A) S x=x$, para $x \in X$ e $S(\lambda I-A) x=x$, para $x \in \mathcal{D}(A)$. 
Lema 2.2 Seja $\gamma:[0, \infty) \rightarrow \mathrm{C}$ uma função com as seguintes propriedades:

1. $\gamma(t+s)=\gamma(t) \gamma(s)$, para todo $t, s \in[0, \infty)$.

2. $\gamma(0)=1$.

3. Existe $t_{0}>0$ tal que $\gamma\left(t_{0}\right) \neq 0$.

4. $\gamma(t)$ continua para $t>a \geq 0$.

Então existe $\lambda \in \mathrm{C}$ tal que $\gamma(t)=e^{\lambda t}$, para $t \geq 0$.

Prova- Temos que $|\gamma(t)|>0$, para todo $t \geq 0$. Tomando a função

$$
f(t)=\log |\gamma(t)|
$$

concluímos que

$$
f(t)=\alpha t \quad \text { para } t>a(\alpha \in \mathbf{R})
$$

Ou seja,

$$
|\gamma(t)|=e^{\alpha t} \text { para } t>a
$$

Consideremos a função

Esta função satisfaz:

$$
\chi(t)=\frac{\gamma(t)}{|\gamma(t)|}
$$

1. $\chi(t+s)=\chi(t) \chi(s)$, para todo $t, s \in[0, \infty)$.

2. $\chi(0)=1$.

3. $\chi(t)$ é contínua para $t>a$.

4. $|\chi(t)|=1$.

Mostremos que $\chi(t)$ é diferenciável para $t \geq a$. Para isso, primeiramente mostraremos que $\chi(t)$ é diferenciável em $t=a$. De fato,

$$
\begin{aligned}
\frac{\chi(\delta+a)-\chi(a)}{\delta} \int_{a}^{\gamma+a} \chi(s) d s & =\frac{1}{\delta} \int_{a}^{\gamma+a} \chi(s+\delta+a) d s-\frac{1}{\delta} \int_{a}^{\gamma+a} \chi(s+a) d s \\
& =\frac{1}{\delta} \int_{\delta+2 a}^{\gamma+\delta+a} \chi(s) d s-\frac{1}{\delta} \int_{2 a}^{\gamma+2 a} \chi(s+a) d s \\
& =\frac{1}{\delta} \int_{\gamma+2 a}^{\gamma+\delta+2 a} \chi(s) d s-\frac{1}{\delta} \int_{2 a}^{\delta+2 a} \chi(s+a) d s
\end{aligned}
$$


Da continuidade de $\chi(t)$ para $t>a$ podemos escolher $\gamma \in[0, \infty)$ tal que

$$
\int_{a}^{\gamma+a} \chi(s) d s \neq 0
$$

Além disso, o limite do lado direito da equação acima existe quando $\delta \rightarrow 0$. Logo, a derivada de $\chi(t)$ existe em $t=a$, isto é,

$$
\left.\frac{d}{d t} \chi(t)\right|_{t=a}=i \beta_{1}
$$

Para $t>a$,

$$
\frac{\chi(t+\delta)-\chi(t)}{\delta}=\chi(t-a) \frac{\chi(a+\delta)-\chi(a)}{\delta} .
$$

Portanto, existe limite quando $\delta \rightarrow 0$, ou seja, $\chi(t)$ é diferenciável para $t \geq a$ e

$$
\dot{\chi}(t)=\chi(t-a) i \beta_{1} \text {. }
$$

Determinar $\chi(t)$ corresponde resolver a equação diferencial acima. Notemos que

$$
\chi(t-a)=\frac{\chi(t-a) \chi(a)}{\chi(a)}=\frac{\chi(t)}{\chi(a)} .
$$

Logo, a equação acima se reduz a

$$
\dot{\chi}(t)=\frac{i \beta_{1}}{\chi(a)} \chi(t) .
$$

A solução desta equação diferencial é dada por

$$
\chi(t)=k \exp \left(\frac{i \beta_{1}}{\chi(a)}\right)(t-a)
$$

para $t \geq a, \operatorname{com} k=\chi(a)$. Chamando $\beta=\beta_{1} / \chi(a)$ temos

$$
\chi(t)=k \exp (i \beta(t-a)) \text {. }
$$

Obsertemos que $\beta \in \mathbf{R}$. Podemos dar uma expressão para $\gamma(t)$, para $t \geq a$. Sabendo que

$$
\gamma(t)=\chi(t)|\gamma(t)|, \text { para } t \geq 0
$$

temos que

$$
\gamma(t)=k e^{-i \beta a} e^{\lambda t}, \text { para } t \geq a
$$

onde $\lambda=\alpha+i \beta$.

Com estas informações podemos estender a caracterização de $\gamma(t)$ para $t \geq 0$. Consideremos $0<t<a$. Então

$$
\gamma(t+a)=k e^{-i \beta a} e^{\lambda(t+a)}=k e^{\alpha a} e^{\lambda t}
$$


e

$$
\gamma(a)=k e^{\alpha a} .
$$

Portanto, $\gamma(t)=e^{\lambda t}$ para $0<t<a$. Resumindo, a função $\gamma(t)$ está assim definida:

$$
\gamma(t)=\left\{\begin{array}{ll}
1 & \text { se } t=0 \\
e^{\lambda t} & \text { se } 0<t<a \\
k e^{-i \beta a} e^{\lambda t} & \text { se } t \geq a
\end{array} .\right.
$$

Da diferenciabilidade de $\chi(t)$ em $t=a$ temos que $k=e^{i \beta a}$. Portanto, $\gamma(t)=e^{\lambda t}$, para $t \geq a$.

Teorema 2.24 Seja $T(t), t \geq 0$, um $C_{0}$-semigrupo eventualmente contínuo. Então o teorema espectral é válido, ou seja,

$$
\sigma(T(t)) \backslash\{0\}=e^{t \sigma(A)}, \text { para } t \geq 0 .
$$

Prova- Resta apenas verificar a inclusão

$$
\sigma(T(t)) \backslash\{0\} \subset e^{t \sigma(A)}, \text { para } t \geq 0 .
$$

Consideremos a subálgebra $\mathcal{B}$ de $L(X)$ definida no Teorema (2.22). Pelo Teorema (2.23) temos que

$$
\sigma(B)=\{B(\mathcal{M}): M \in \mathcal{M}\} .
$$

Tomemos $0 \neq c \in \sigma\left(T\left(t_{0}\right)\right)$. Logo,

$$
c=T\left(t_{0}\right)(M), \text { para algum } M \in \mathcal{M} .
$$

Consideremos a função $\gamma:[0, \infty) \rightarrow \mathbf{C}$ tal que

$$
\gamma(t)=T(t)(M)
$$

$\Lambda$ função acima está nas condiçôes do Lema (2.2). Portanto, $\gamma(t)=e^{\lambda t}$, para algum $\lambda \in \mathbf{C}$. Mostremos que $\lambda \in \sigma(A)$. Seja $\Re(z)>\omega$. Então

$$
R(z, A) x=\int_{0}^{\infty} e^{-z t} T(t) x d t, \text { para } x \in X
$$

e

$$
T(a) R(z, A)=\int_{0}^{\infty} e^{-z t} T(a+t) d t
$$

onde a convergência é na norma do operador. Ainda

$$
(T(a) R(z, A))(M)=e^{\lambda a} R(z, A)(M) .
$$


Por outro lado, mostremos que $(T(a) R(z, A))(M)=\int_{0}^{\infty} e^{-z t} e^{(a+t) \lambda} d t$.

Basta mostrar que $\int_{0}^{\infty} e^{-z t} e^{(a+t) \lambda} d t \in \sigma(T(a) R(z, A)+M)$. De fato,

$$
\begin{aligned}
{[T(a) R(z, A)+M] } & -\int_{0}^{\infty} e^{-z t} e^{(a+t) \lambda} d t(I+M) \\
& =\left[T(a) R(z, A)-\int_{0}^{\infty} e^{-z t} e^{(a+t) \lambda} d t\right]+M \\
& =\int_{0}^{\infty} e^{-z t}\left[T(a+t)-e^{\lambda(a+t)} I\right] d t+M \\
& =\int_{0}^{\infty} e^{-z t}[T(a+t)-T(a+t)(M) I] d t+M=M
\end{aligned}
$$

Portanto,

$$
R(z, A)(M)=\int_{0}^{\infty} e^{(\lambda-z) t} d t=(z-\lambda)^{-1}
$$

Ou seja, $(z-\lambda)^{-1} \in \sigma(R(z, A))$. Pelo Lema (2.1) temos $\lambda \in \sigma(A)$. Concluímos que $c \in e^{t_{0} \sigma(A)}$.

O Corolário abaixo fornece classes importantes de semigrupos, todos eventualmente contínuos, onde o teorema espectral é válido.

Corolário 2.7 O teorema espectral,

$$
\sigma(T(t)) /\{0\}=e^{t \sigma(A)}, t \geq 0
$$

vale para cada uma das seguintes classes de semigrupos fortemente contínuos:

1. semigrupos eventualmente compactos.

2. semigrupos eventualmente diferenciáveis.

3. semigrupos analíticos.

4. semigrupos uniformemente contínuos.

A limitação do resultado obtido no Teorema (2.24) reside no fato de que não confrecemos condições sobre o gerador ou sobre o operador resolvente do gerador de um semigrupo que nos permita saber quando um semigrupo é eventualmente contínuo. $\mathrm{O}$ resultado a seguir nos dá uma condição necessária para um gerador infinitesimal $A$ gerar um $C_{0^{-}}$-semigrupo eventualmente contínuo. 
Teorema 2.25 Sejam $T(t), t \geq 0$, um $C_{0}$-semigrupo e A seu gerador infinitesimal. Se $T(t)$ é eventualmente contínuo para $t_{0}=0$, entâo existe um função $\psi:[0, \infty) \rightarrow[0, \infty)$ tal que

$$
\rho(A) \supset\{\lambda: \lambda=\sigma+i \tau,|\tau| \geq \psi(|\sigma|)\},
$$

$e$

$$
\lim _{|\tau| \rightarrow \infty}\|R(\sigma+i \tau, A)\|=0
$$

Prova- Ver Pazy [9, pag. 50]. 


\section{Capítulo 3}

\section{Teoria Espectral para Espaços de Hilbert}

Como vimos no capítulo anterior, para semigrupos eventualmente contínuos vale o teorema espectral. Esse resultado é conhecido desde 1958, com a publicação de Hille-Phillps [7]. Porém não temos uma caracterização completa, no seguinte sentido, ainda ficamos com uma pergunta: será que somente para os semigrupos eventualmente contínuos definidos sobre um espaço de Banach vale o teorema espectral? Em 1978, Gearhart [3] publicou um resultado onde obteve uma caracterização do espectro para semigrupos de contrações definidos sobre um espaço de Hilbert. Porém, sua prova é complicada e longa. Prüss [10] e Herbst [6] estenderam e simplificaram este resultado. Ambos conseguiram a mesma caracterização obtida por Gearhart para o caso de $C_{0}$-semigrupos definidos sobre um espaço de Hilbert.

O objetivo deste capítulo é expôr os resultados obtidos por Prüss. O resultado diz que existem dois modos de obtermos pontos no espectro de $T(t)$ :

1. por pontos provenientes do espectro de $A$, via função exponencial,

2. por pontos do resolvente de $A$ onde não há limitação uniforme da função resolvente nas retas $\Re(z)=$ constante.

Além disso, no segundo caso os pontos estarão no espectro contínuo de $T(t)$.

A seguir, analisamos dois semigrupos particulares. $\mathrm{O}$ primeiro foi apresentado pela primeira vez por Hille-Phillips em [7]. Porém foi realmente entendido, dentro do contexto de teoria espectral, depois da publicação do resultado de Gearhart. 
Analisando o particular semigrupo vemos que é um exemplo do teorema obtido por Gearhart. E surge-nos uma nova questão: é possível obter o mesmo resultado para um espaço de Banach? O segundo exemplo nos mostra que não. Fíca então a questão: quais propriedades adicionais sobre o resolvente de $A$ são necessárias para conseguirmos uma caracterização de $\sigma(T(t))$ ?

\subsection{Soluções Periódicas}

O trabalho de Prüss para simplificar e estender o resultado de Gearhart utiliza a relação entre o espectro de $T(t)$ e as soluções periódicas de um problema do valor inicial não homogêneo.

Seja $X$ um espaço de Banach. Consideremos $T(t), t \geq 0$, um $C_{0^{-}}$semigrupo e $A$ seu gerador infinitesimal. Dado $f \in L^{1}([0, \omega], X)$, uma função $u \in C([0, \omega], X)$ é chamada uma solução fraca de

$$
\dot{u}(t)=A u(t)+f(t)
$$

sobre $[0, \omega]$, com condição inicial $u_{0} \in X$, se

$$
u(t)=\omega(t) u_{0}+\int_{0}^{t} T(t-s) f(s) d s
$$

é verdadeira sobre $[0, \omega]$. Ainda, $u(t)$ satisfaz $(3.1)$, para cada $t \in[0, \omega]$ se, e somente se $u \in C^{1}([0, \omega], X)$. Se $u(t)$ é uma solução fraca sobre $[0, \omega]$ tal que $u(0)=u(\omega)$, então $u(t)$ pode ser continuamente estendida por periodicidade para todo $t \in \mathbf{R}$, ou seja, $u(t)$ é uma solução fraca $\omega$-periódica sobre $\mathbf{R}$ desde que $f(t)$ também possa ser estendida periodicamente. Assim, se $u(0)=u(\omega)$, chamaremos $u(t)$ um solução fraca de (3.1) sobre $[0, \omega], \omega$-periódica .

Lema 3.1 Sejam $T(t), t \geq 0$, um $C_{0}$-semigrupo e $A$ seu gerador infinitesimal. $\mu=e^{\lambda \omega} \in \rho(T(\omega))$ se, e somente se $1 \in \rho(S(1))$ onde $S(t), t \geq 0$, é o $C_{0^{-}}$ semigrupo $e^{-\lambda \omega t} T(\omega t)$, cujo gerador infinitesimal é $B=(A-\lambda) \omega$.

Portanto, é suficiente considerarmos $1 \in \rho(T(1))$ e soluções 1-periódicas.

Teorema 3.1 Sejam $X$ um espaço de Banach, $T(t), t \geq 0$, um $C_{0}$-semigrupo e $A$ seu gerador infinitesimal. Então $1 \in \rho(T(1))$ se, e somente se para qualquer $f \in C([0,1], X),(3.1)$ admite uma única solução fraca 1-periódica. 
Prova- Se $1 \in \rho(T(1))$, então $I-T(1)$ é inversível. Considerando o PVI

$$
\left\{\begin{array}{l}
\dot{u}(t)=A u(t)+f(t) \\
u(0)=u_{0}
\end{array}\right.
$$

para $t \in[0,1]$, onde

$$
u(0)=u_{0}=(I-T(1))^{-1} \int_{0}^{1} T(1-s) f(s) d s,
$$

então uma solução fraca para o PVI acima é dada por

$$
u(t)=T(t) u_{0}+\int_{0}^{t} T(t-s) f(s) d s .
$$

É fácil ver que $u(t)$ é 1-periódica e ainda mais, é a única solução fraca da equação (3.1). Reciprocamente, definamos a aplicação

$$
K: C([0,1], X) \rightarrow C([0,1], X)
$$

dada por $(K f)(t)=u(t)$, onde $u(t)$ é a única solução fraca 1-periódica de (3.1). A aplicação $K$ é um operador linear sobre $C([0,1], X)$. Devido a unicidade da solução fraca 1-periódica e pelo Teorema do Gráfico Fechado [11], podemos concluir que $K$ é um operador limitado. Tomando, em particular, $f(t)=T(t) x$ para $x \in X$ e definindo $S x=(K f)(0)$, temos que $S$ é um operador linear limitado sobre $X$ com a propriedade:

$$
(I-T(1))(S x+x)=x \text { para todo } x \in X .
$$

Ou seja, $I-T(1)$ é sobrejetor e, mais uma vez, a unicidade da solução fraca 1-periódica nos permite concluir que $I-T(1)$ é injetor. Portanto, $(I-T(1))$ é inversivel e $R(1, T(1))=S+1$. Ou seja, $1 \in \rho(T(1))$.

Lema 3.2 Seja $X$ um espaço de Hilbert. Se $\left(\varphi_{\alpha}\right)_{\alpha \in A}$ é um sistema ortonormal completo em $X$ e $f_{n}(t)=e^{2 \pi i n t}$, para $n \in \mathbf{Z}$ então o conjunto $\left(f_{\beta}\right)_{\beta \in \mathbf{Z} \times A}$, onde $f_{\beta}=f_{n} \varphi_{\alpha}$, é um sistema ortonormal completo em $L_{2}([0,1], X)$.

Prova- Seja $f$ tal que $<f, f_{\beta}>=0$ em $L_{2}([0,1], X)$. Então, $<g_{\alpha}, f_{n}>=0$ onde $g_{\alpha}(t)=<f(t), \varphi_{\alpha}>$ em $L_{2}([0,1])$. Como $\left(f_{n}\right)_{n \in Z}$ constitui um sistema ortonormal completo em $L_{2}([0,1])$, temos $g_{\alpha} \equiv 0$, para cada $\alpha \in A$. Pelo fato de $A$ também ser um sistema ortonormal completo em $X$, temos que $f(t)=0$, para todo $t \in[0,1]$. Pelo Teorema da Base [1], temos que $\left(f_{\beta}\right)_{\beta \in \mathbf{Z} \times A}$ constitui um sistema ortonormal completo. 
Lema 3.3 Sejam $X$ um espaço de Hilbert e $f \in L_{2}([0,1], X)$. Então

$$
\|f\|_{2}^{2}=\sum_{n \in \mathbb{Z}}\left|g_{n}\right|^{2}
$$

onde $g_{n}=\int_{0}^{1} e^{-2 \pi i n s} f(s) d s$. Além disso,

$$
f(\cdot)=\lim _{n \rightarrow \infty} \sum_{k=-n}^{n} g_{n} e^{2 \pi i n(\cdot)},
$$

onde o limite é em $L_{2}([0,1], X)$.

Prova- Do lema anterior e do Teorema da Base podemos concluímos que

$$
\begin{aligned}
\|f\|^{2} & =\sum_{\beta \in \mathbf{Z} \times \boldsymbol{A}}\left|<f, f_{\beta}>\right|^{2}=\sum_{n \in \mathbf{Z}} \sum_{\alpha \in A}\left|\int_{0}^{1}<f(s) e^{-2 \pi i n s}, \varphi_{\alpha}>\right|^{2} d s \\
& =\sum_{n \in \mathbf{Z}}\left|g_{n}\right|^{2}
\end{aligned}
$$

e

$$
\begin{aligned}
f & =\sum_{\beta \in \mathbf{Z} \times A}<f, f_{\beta}>f_{\beta}=\sum_{n=-\infty}^{\infty}\left[\sum_{\alpha \in A}<f \overline{f_{n}(\cdot)}, \varphi_{\alpha}>\varphi_{\alpha}\right] f_{n}(\cdot) \\
& =\sum_{n=-\infty}^{\infty} g_{n} f_{n}(\cdot),
\end{aligned}
$$

onde $g_{n}=\int_{0}^{1} e^{-2 \pi i n s} f(s) d s$.

Teorema 3.2 Sejam $X$ um espaço de Hilbert e $T(t), t \geq 0$, um $C_{0}$-semigrupo gerado por $A$. Então $1 \in \rho(T(1))$ se, e somente se, $2 \pi i n \in \rho(A)$ para todo $n \in \mathbf{Z} e \sup \{\|R(2 \pi i n, A)\|: n \in \mathbf{Z}\}=M<\infty$.

Prova- A primeira afirmativa da condição necessária é consequência imediata do Lema (1.1). A limitação uniforme de $\|R(2 \pi i n, A)\|$ segue do fato que

$$
R(2 \pi i n, A) x=R(1, T(1)) \int_{0}^{1} e^{-2 \pi i n s} T(s) x d s,
$$

para todo $x \in X$.

Reciprocamente, queremos provar que $1 \in \rho(T(1))$. Pelo Teorema (3.1) basta verificar que para todo $f \in C([0,1], X)$, a equação (3.1) admite precisamente 
uma única solução fraca 1-periódica. Assim, seja $f \in C([0,1], X)$. Pelo Lema (3.3) temos que

$$
\|f\|_{2}^{2}=\sum_{n=-\infty}^{\infty}\left|f_{n}\right|^{2}, \text { e } f(\cdot)=\lim _{n \rightarrow \infty} \sum_{k=-n}^{n} g_{n} e^{2 \pi i n(\cdot)}
$$

onde $g_{n}=\int_{0}^{1} e^{-2 \pi i n s} f(s) d s$.

Se $u(t)$ é uma solução fraca 1-periódica de (3.1), então temos que os coeficientes da série de Fourier de $u$ são

$$
u_{n}=(2 \pi i n-A)^{-1} g_{n}
$$

De fato,

$$
\begin{aligned}
(2 \pi i n-A) u_{n} & =(2 \pi i n-A) \int_{0}^{1} e^{-2 \pi i n t} T(t) u_{0} d t \\
& +(2 \pi i n-A) \int_{0}^{1} \int_{0}^{t} e^{-2 \pi i n t} T(t-s) f(s) d s d t \\
& =-T(1) u(0)+u(0)-\int_{0}^{1} T(1-s) f(s) d s+\int_{0}^{1} e^{-2 \pi i n s} f(s) d s
\end{aligned}
$$

Ou seja, provamos que há no máximo uma solução fraca 1-periódica. Tomemos

$$
u_{N}(t)=\sum_{k=-N}^{N} e^{2 \pi i k t} u_{k}
$$

Logo, $u_{N} \in C^{1}-([0,1], X), u_{N}$ é uma função 1-periódica e satisfaz

$$
u_{N}(t)=T(t) u_{N}(0)+\int_{0}^{t} T(t-s) f_{N}(s) d s,
$$

onde $f_{N}(t)=\sum_{k=-N}^{N} e^{2 \pi i k t} f_{k}$. Como

$$
\sum_{k=-N}^{S}\left|u_{n}\right|^{2} \leq M^{2}\|f\|_{2}^{2}
$$

pela igualdade de Parseval, temos que $u(t)=\sum_{n=-\infty}^{\infty} u_{n} e^{2 \pi i n t} \in L_{2}([0,1], X)$ e $u_{N}$ converge para $u$ em $L_{2}([0,1], X)$.

Mostremos que $u$ é solução fraca 1-periódica de (3.1). Primeiramente, analisemos algumas convergências

$$
\int_{0}^{t} T(t-s) f_{N}(s) d s \rightarrow \int_{0}^{t} T(t-s) f(s) d s
$$


quando $N \rightarrow \infty$ e uniformemente para $t \in[0,1]$. Isto segue da desigualdade

$$
\begin{aligned}
\mid \int_{0}^{t} T(t-s) f_{N}(s) d s & -\int_{0}^{t} T(t-s) f(s) d s\left|\leq \int_{0}^{t}\right| T(t-s) \| f_{N}(s)-f(s) \mid d s \\
& \leq \sup \{|T(u)|: 0 \leq u \leq 1\}\left\|f_{N}-f\right\|_{2} .
\end{aligned}
$$

Além disso,

$$
u_{N}(1)=T(1) u_{N}(0)+\int_{0}^{1} T(1-s) f_{N}(s) d s
$$

Portanto,

$$
(I-T(1)) u_{N}(0)=\int_{0}^{1} T(1-s) f_{N}(s) d s \rightarrow \int_{0}^{1} T(1-s) f(s) d s .
$$

Mutiplicando por $T(1-t)$ e integrando sobre $[0,1]$ a equação (3.3) temos

$$
T(1) u_{N}(0)=\int_{0}^{1} T(1-s) u_{N}(t) d t-\int_{0}^{1} T(1-t) \int_{0}^{t} T(t-s) f_{N}(s) d s .
$$

Logo, $T(1) u_{N}(0)$ converge quando $N \rightarrow \infty$. Desse modo, $u_{N}(0)$ converge quando $N \rightarrow \infty, u_{N} \rightarrow u$ em $C([0,1], X)$ e $u(t)$ é uma solução fraca 1-periódica de (3.1).

O teorema a seguir dá uma caracterização do resolvente de um $C_{0}$-semigrupo definido sobre um espaço de Hilbert, cuja demonstração é imediata a partir do Teorema (3.2).

Teorema 3.3 Sejam $X$ um espaço de Hilbert e $T(t), t \geq 0$, um $C_{0}$-semigrupo em $X$. São equivalentes

1. $0 \neq \mu=e^{\lambda t} \in \rho(T(t))$.

2. $\lambda+2 \pi i n / t \in \rho(A)$, para todo $n \in \mathbf{Z}$ e $\sup _{n} \in \mathbf{Z}\|R(\lambda+2 \pi i n / t, A)\|<\infty$.

\subsection{Um exemplo: O Semigrupo das Integrais Fracionárias}

De 1948 até 1978 o semigrupo das integrais fracionária era visto somente como um contra exemplo da validade do Teorema Espectral. Mesmo depois de 1983, o Teorema de Prüss não era aplicado a este exemplo. Em 1987, Henry [5] 
obteve a interpretação do semigrupo que colocou-o como exemplo da situação descrita no Teorema (3.3).

Por diversas vezes serão utilizados resultados a respeito da função gama que podem ser encontrados em Conway [2] ou Henry [4].

Consideremos $f:(0,1) \rightarrow \mathrm{C}$ tal que o suporte de $f$ é compacto e $t>0$, definimos

$$
J^{t} f(x)=\int_{0}^{x} \frac{(x-y)^{t-1}}{\Gamma(t)} f(y) d y
$$

Para quaisquer $t, s \geq 0$ temos

$$
\begin{aligned}
J^{t}\left(J^{s} f\right)(x) & =\int_{0}^{x} \frac{(x-y)^{t-1}}{\Gamma(t)}\left[\int_{0}^{y} \frac{(y-z)^{s-1}}{\Gamma(s)} f(z) d z\right] d y \\
& =\int_{0}^{x}\left[\int_{z}^{x} \frac{(x-y)^{t-1}(y-z)^{s-1}}{\Gamma(t) \Gamma(s)} d y\right] f(z) d z \\
& =\int_{0}^{x} \frac{(x-z)^{t+s-1}}{\Gamma(t+s)} f(z) d z=J^{t+s} f(x)
\end{aligned}
$$

Lema 3.4 A definição de $J^{t}$ faz sentido para qualquer número complexo $t$, tal que $\Re(t)>0$.

Prova- Para $t \in \mathbf{C}$ tal que $\Re(t)>0$, a função $g(y)=(x-y)^{t-1}$ é integravél em $[0, x]$. Isto segue do fato que

$$
\left|\int_{0}^{x}(x-y)^{t-1} d y\right| \leq \int_{0}^{x}(x-y)^{\Re(t)-1} d y
$$

onde esta última integral é convergente.

Proposição 3.1 Dada uma função $f \in L_{2}(0,1)$, temos que $J^{t} f \in L_{2}(0,1)$.

Prova- Definamos

$$
K_{t}(u)=\left\{\begin{array}{ccc}
u^{t-1} / \Gamma(t) & \text { se } & u>0 \\
0 & \text { se } & u<0
\end{array} .\right.
$$

Se $0<x<1, \Re(t)>0$ e $f \in C_{0}^{\infty}(0,1)$ então

$$
\begin{aligned}
\left|J^{t} f(x)\right| & =\left|\int_{0}^{1} K_{t}(x-y) f(y) d y\right| \\
& \leq\left(\int_{0}^{1}\left|K_{t}(x-y)\right| d y\right)^{\frac{1}{2}}\left(\int_{0}^{1}\left|K_{t}(x-y)\right||f(y)|^{2} d y\right)^{\frac{1}{2}}
\end{aligned}
$$


Como

$$
\int_{0}^{1}\left|K_{t}(x-y)\right| d y \leq \int_{0}^{1}\left|K_{t}(u)\right| d u \text { e } \int_{0}^{1}\left|K_{t}(x-y)\right| d x \leq \int_{0}^{1}\left|K_{t}(u)\right| d u
$$

concluímos que

$$
\int_{0}^{1}\left|J^{t} f(x)\right|^{2} d x \leq\left(\int_{0}^{1}\left|K_{t}(u)\right| d u\right)^{2} \int_{0}^{1}|f(u)|^{2} d u
$$

Chamando

$$
B(t)=\int_{0}^{1}\left|K_{t}(u)\right| d u=\int_{0}^{1} \frac{u^{\Re(t)-1}}{|\Gamma(t)|} d u=\frac{1}{\Re(t)|\Gamma(t)|}
$$

temos que

$$
\left\|J^{t} f\right\|_{2} \leq B(t)\|f\|_{2} \text { para todo } f \in C_{0}^{\infty}(0,1) .
$$

Portanto, se $f \in L_{2}(0,1)$ então $j^{t} f \in L_{2}(0,1)$.

Corolário 3.1 Os operadores $J^{t}, \Re(t)>0$, formam uma familia de operadores lineares limitados definidos sobre $L_{2}(0,1)$.

Proposição 3.2 Para cada $f \in L_{2}(0,1)$, a função que leva $t$ em $J^{t} f$ é analítica para todo $t \in \mathbf{C}$ tal que $\Re(t)>0$.

Prova- Dado $f \in L_{2}(0,1)$, basta provarmos que a função complexa $\xi\left(J^{t}(f)\right)$ é analítica para cada $\xi \in L_{2}(0,1)^{\prime}$. Sabemos que existe $g \in L_{2}(0,1)$ tal que

$$
\xi(f)=\int_{0}^{1} f(t) \overline{g(t)} d t .
$$

Portanto,

$$
\xi\left(J^{t}(f)\right)=\frac{1}{\Gamma(t)} \int_{0}^{1} \int_{0}^{u}(u-y)^{t-1} f(u) \overline{g(u)} d y d u .
$$

Considerando $F(u, t)=\int_{0}^{u}(u-y)^{t-1} f(u) \overline{g(u)} d y$, temos

1. $u \rightarrow F(u, t)$ é integrável para cada $t$.

2. $|\partial F / \partial t| \leq h(u)$ onde $h$ é uma função integrável.

Com estes fatos concluímos que $t \rightarrow \xi\left(J^{t}(f)\right)$ é analítica para todo $\xi \in L_{2}(0,1)^{\prime}$. 
Corolário 3.2 Dodo $f \in L_{2}(0,1)$ temos que $J^{t}\left(J^{s} f\right)=J^{t+s} f$ para $\Re(t)>0 e$ $\Re(s)>0$.

Prova- Desde que a igualdade ocorre para $t, s>0$, do príncipio da continuação analítica segue o corolário.

Proposição 3.3 A função $t \rightarrow J^{t} f$ é contínua quando $t \rightarrow 0$ em qualquer setor $\{t \in \mathrm{C}:|\arg (t)| \leq \pi / 2-\epsilon<\pi / 2\}$.

Prova- Basta observarmos que para $f \in C_{0}^{\infty}(0,1)$ temos

$$
J^{t} f(x)=f(x)+\left[\frac{x^{t}}{\Gamma(1+t)}-1\right] f(x)-\frac{t}{\Gamma(1+t)} \int_{0}^{x}(x-y)^{t} \frac{f(x)-f(y)}{x-y} d y
$$

e que $\Gamma(1+t) \rightarrow 1$ quando $t \rightarrow 0$.

Temos, assim, um $C_{0}$-semigrupo em $\Re(t)>0$ e, em particular, sobre eixo real $t \geq 0$. Antes de prosseguirmos, mostraremos que em certos casos é possível estender a função $t \rightarrow J^{t} f$ para todo plano complexo.

Proposição 3.4 Seja $f \in C_{0}^{\infty}(0,1)$. É possível estender $J^{t} f$ para $\Re(t)<0$ de modo que $J^{-j} f(x)=f^{(j)}(x)$, para $j \in \mathbf{N}$.

Prova- Tomando $f \in C_{0}^{\infty} \cdot(0,1)$ temos

$$
\begin{aligned}
J^{t} f(x) & =\int_{0}^{x} \frac{(x-y)^{t-1}}{\Gamma(t)}\left[f(y)-\sum_{j=0}^{N} \frac{f^{(j)}(x)}{j !}(y-x)^{j}\right] d y \\
& +\int_{0}^{x} \frac{(x-y)^{t-1}}{\Gamma(t)} \sum_{j=0}^{N} \frac{f^{(j)}(x)}{j !}(y-x)^{j} d y \\
& =\int_{0}^{x} \frac{(x-y)^{t-1}}{\Gamma(t)}\left[f(y)-\sum_{j=0}^{N} \frac{f^{(j)}(x)}{j !}(y-x)^{j}\right] d y \\
& +\sum_{j=0}^{N} \frac{f^{(j)}(x)}{j ! \Gamma(t)}(-1)^{t-1} \int_{0}^{x}(y-x)^{t+j-1} d y \\
& =\int_{0}^{x} \frac{(x-y)^{t-1}}{\Gamma(t)}\left[f(y)-\sum_{j=0}^{N} \frac{f^{(j)}(x)}{j !}(y-x)^{j}\right] d y+\sum_{j=0}^{N} \frac{f^{(j)}(x)}{j !} \frac{(-1)^{j} x^{t+j}}{(t+j) \Gamma(t)}
\end{aligned}
$$


Mostremos que $J^{t} f(x)$ faz sentido para $\Re(t)<0$ e que $\lim _{t \rightarrow-N} J^{t} f(x)=f^{N}(x)$ para cada $N \in N$. Seja

$$
g^{t}(x)=\int_{0}^{x} \frac{(x-y)^{t-1}}{\Gamma(t)}\left[f(y)-\sum_{j=0}^{N} \frac{f^{(j)}(x)}{j !}(y-x)^{j}\right] d y+\sum_{j=0}^{N} \frac{f^{(j)}(x)}{j !} \frac{(-1)^{j} x^{t+j}}{(t+j) \Gamma(t)} .
$$

Então

$$
\begin{aligned}
\mid \int_{0}^{r} \frac{(x-y)^{t-1}}{\Gamma(t)}[f(y) & \left.-\sum_{j=0}^{N} \frac{f^{(j)}(x)}{j !}(y-x)^{j}\right] d y \mid \\
& \leq \sup _{0 \leq \tau \leq x} \frac{\left|f^{(N+1)}(\tau)\right|}{(N+1) !} \int_{0}^{\tau} \frac{(x-y)^{\Re(t)+N}}{|\Gamma(t)|} d y \\
& =\frac{\left(x^{\Re(t)+N+1}-(x-r)^{\Re(t)+N+1}\right)}{(\Re(t)+N+1)|\Gamma(t)|} \sup _{0 \leq \tau \leq x} \frac{\left|f^{(N+1)}(\tau)\right|}{(N+1) !} .
\end{aligned}
$$

Restringindo ao semiplano $\Re(t)>-(N+1)$ obtemos que $o$

$$
\left.\lim _{r \rightarrow x}\left|\int_{0}^{r} \frac{(x-y)^{t-1}}{\Gamma(t)}\right| f(y)-\sum_{j=0}^{N} \frac{f^{(j)}(x)}{j !}(y-x)^{j}\right] d y \mid
$$

existe, a convergência é absoluta e, para conjuntos compactos desse semiplano a convergência é uniforme. Temos que $g^{t}$ é analítica e $g^{t}=J^{t} f$ para. $\Re(t)>$ 0 . Definindo $g^{t}=J^{t} f$ para $\Re(t)<N+1$ temos que $J^{t} f$ pode ser estendida analiticamente em $\mathbf{C}-\{t: t \leq 0\}$. Assim, $t=-j$, para $j=0,1,2, \cdots, N$, são os possiveis polos de $J^{t} f$. Mostremos que $J^{t} f$ também está definida nestes casos. Sendo

$$
\frac{1}{(t+j) \Gamma(t)}=\frac{\Gamma(t) \operatorname{sen} \pi t}{\pi(t+j)}
$$

concluímos que

$$
\lim _{t \rightarrow-k} \frac{1}{(t+j) \Gamma(t)}=\left\{\begin{array}{ccc}
0 & \text { se } & k \neq j \\
(-1)^{j} j ! & \text { se } & k=j
\end{array} .\right.
$$

Desse modo,

$$
\lim _{t \rightarrow-N} \sum_{j=0}^{N} \frac{f^{(j)}(x)}{j !} \frac{(-1)^{j} x^{t+j}}{(t+j) \Gamma(t)}=f^{(N)}(x)
$$

Além disso, a desigualdade

$$
\begin{aligned}
\mid \int_{0}^{x} \frac{(x-y)^{t-1}}{\Gamma(t)}[f(y) & \left.-\sum_{j=0}^{N} \frac{f^{(j)}(x)}{j !}(y-x)^{j}\right] d y \mid \\
& \leq \frac{x^{\Re(t)+j+1}}{(\Re(t)+N+1)|\Gamma(t)|} \sup _{0 \leq r \leq x} \frac{\left|f^{(N+1)}(\tau)\right|}{(N+1) !}
\end{aligned}
$$


nos mostra que

$$
\lim _{t \rightarrow-N} \int_{0}^{x} \frac{(x-y)^{t-1}}{\Gamma(j)}\left[f(y)-\sum_{j=0}^{N} \frac{f^{(j)}(x)}{j !}(y-x)^{j}\right] d y=0 .
$$

Portanto, $\lim _{t \rightarrow-j} J^{t} f(x)=f^{(j)}(x)$, para $j \in \mathrm{N}$.

Com as informações já obtidas podemos determinar o espectro de $A$, o gerador infinitesimal de $J^{t}, t \geq 0$, mesmo sem conhecê-lo.

Teorema 3.4 Seja A o gerador infinitesimal de $J^{t}, t \geq 0$. Então $\sigma(A)$ é vazio.

Prova- Da Proposição (3.1) e da definição de raio espectral obtemos

$$
r\left(J^{t}\right)=\lim _{n \rightarrow \infty}\left\|J^{n t}\right\|^{\frac{1}{n}} \leq \lim _{n \rightarrow \infty} \frac{1}{(n t \Gamma(n t))^{\frac{1}{n}}}=\lim _{n \rightarrow \infty} \frac{1}{\Gamma(n t+1)^{\frac{1}{n}}} .
$$

Como

$$
\log \Gamma(z+1)=(z+1 / 2) \log z-z+\log \sqrt{2 \pi}+O(1 /|z|)
$$

temos

$$
\frac{1}{\Gamma(n t+1)^{\frac{1}{n}}}=\frac{1}{\left(\sqrt{n t}(n t)^{n t} e^{-n t} \sqrt{2 \pi} e^{O\left(\frac{1}{n t}\right)}\right)^{\frac{1}{n}}}
$$

e portanto,

$$
r\left(J^{t}\right)=0 .
$$

Ou seja, $\sigma\left(J^{t}\right)=\{0\}$. Como a inclusão $e^{\sigma(A) t} \subset \sigma\left(J^{t}\right)$ é sempre verdadeira temos que $\sigma(A)=\emptyset$.

Lema 3.5 Sejam $\xi \in \mathbf{R}$ e $0 \leq \Re(t)<1 / 2$. Então

$$
\int_{0}^{\infty} \frac{u^{t-1} e^{-i \xi u}}{\Gamma(t)} d u=|\xi|^{-t} e^{\mp \pi t / 2}
$$

onde $\mp=-\operatorname{sgn}(\xi)$.

Prova- Faremos apenas o caso $\xi>0$. Consideremos a função complexa $f(z)=$ $z^{t-1} e^{-i \xi z} / \Gamma(t)$ e o contorno fechado simples $\gamma=\gamma_{1}+\gamma_{2}+\gamma_{3}$, onde $\gamma_{1}(s)=s$, para $0 \leq s \leq R ; \gamma_{2}(s)=R e^{-i s}$, para $0 \leq s \leq \pi / 2$ e $\gamma_{3}(s)=i s$, para $0 \leq s \leq R$. 
Pelo Teorema de Cauchy temos que $\int_{\gamma} f=0$ para todo $R>0$. Mostremos que $\lim _{R \rightarrow \infty} \int_{\gamma_{2}} f(z) d z=0$. Isto é imediaio das desigualdades:

$$
\begin{aligned}
\left|\int_{\gamma_{2}} \frac{z^{t-1} e^{-i \xi z}}{\Gamma(t)} d z\right| & \leq \frac{R^{\Re(t)}}{\left.\mid \Gamma \frac{1}{\mid}\right) !} \int_{0}^{\pi / 2} e^{s \Im(t)} e^{-\xi R \operatorname{sen} s} d s \\
& \leq K \frac{\varepsilon^{\Re(t)}}{\Gamma(t) \mid} \int_{0}^{\pi / 2} e^{-\xi R \operatorname{sen} s} d s \leq K \frac{R^{\Re(t)}}{|\Gamma(t)|} \int_{0}^{\pi / 2} e^{-\xi R 2 s / \pi} d s \\
& =\frac{K \pi}{\xi|\Gamma(t)|} R^{\Re(t)-1}\left[1-e^{-\xi R}\right] .
\end{aligned}
$$

Teorema 3.5 O semigrupo $J^{t}$ pode ser definido e é um $C_{0}$-semigrupo no semiplano fechado $\Re(t) \geq 0$.

Prova- Seja $f \in C_{0}^{\infty}(0,1)$. Para todo $t$ complexo podemos definir $J^{t} f$. Consideremos a particular faixa $0 \leq \Re(t)<1 / 2$. Definindo $J^{t} f(x)=0$, para $x<0$ e $J^{t} f(x)=\int_{0}^{1}(x-y)^{t-1} f(y) / \Gamma(t) d y$, para $x \geq 1$, podemos calcular a transformada de Fourier quando $0 \leq \Re(t)<1 / 2$ :

$$
\begin{aligned}
\left(J^{t} f\right)^{\wedge}(\xi) & =\lim _{R \rightarrow \infty} \int_{-R}^{R} e^{-i \xi x} J^{t} f(x) d x \\
& =\lim _{R \rightarrow \infty} \int_{0}^{R} \int_{y}^{R} \frac{(x-y)^{t-1}}{\Gamma(t)} f(y) e^{-i \xi x} d x d y \\
& =\lim _{R \rightarrow \infty} \int_{0}^{1}\left[\int_{0}^{R-y} \frac{u^{t-1} e^{-i \xi u}}{\Gamma(t)} d u\right] e^{-i \xi y} f(y) d y .
\end{aligned}
$$

Pelo Lema (3.5) temos

$$
\left(J^{t} f\right)^{\wedge}(\xi)=|\xi|^{-t} e^{\mp i \pi t / 2} \hat{f}(\xi)
$$

Então

$$
\begin{aligned}
\int_{0}^{\infty}\left|f^{t} f(x)\right|^{2} d x & \leq \frac{1}{2 \pi} e^{\pi|\Im(t)|}\left(\int_{-\infty}^{\infty}|\hat{f}(\xi)|^{2} d \xi+\int_{-1}^{1}\left(|\xi|^{-2 \Re(t)}-1\right)|\hat{f}(\xi)|^{2} d \xi\right) \\
& \leq \frac{1}{2 \pi} e^{\pi|\Im(t)|}\left(\|f\|_{2}^{2}+\|f\|_{2}^{2} \frac{4 \Re(t)}{1-2 \Re(t)}\right) .
\end{aligned}
$$

Portanto,

$$
\left\|J^{t} f\right\|_{2} \leq e^{\pi|\Im(t)| / 2}\left(1+\frac{2 \Re(t)}{\pi(1-2 \Re(t))}\right)^{1 / 2}\|f\|_{2} \leq \frac{e^{\pi|\Im(t)| / 2}}{(1-2 \Re(t))^{1 / 2}}\|f\|_{2} .
$$

Logo, $J^{t} f \in L_{2}(0,1)$ e podemos definir $J^{t} f$, para todo $f \in L_{2}(0,1)$. Ou seja, $J^{t}$ é um semigrupo em $\Re(t) \geq 0$. 
Determinemos o gerador infinitesimal do semigrupo. Para tanto, inicialmente estabelecemos três resultados técnicos que nos auxiliarão na sua determinação.

Lema 3.6 Para $\Re(t) \geq 1$ e $f \in L_{2}(0,1)$ temos

$$
\frac{\partial}{\partial x}\left(J^{t} f(x)\right)=J^{t-1} f(x)
$$

Prova- Seja $f \in C(0,1)$. Então

$$
\begin{aligned}
\frac{1}{t}\left[J^{t} f(x+h)-J^{t} f(x)\right] & =\frac{1}{h} \int_{0}^{x} \frac{(x+h-y)^{t-1}-(x-y)^{t-1}}{\Gamma(t)} f(y) d y \\
& +\frac{1}{h} \int_{x}^{x+h} \frac{(x+h-y)^{t-1}}{\Gamma(t)} f(y) d y
\end{aligned}
$$

Como

$$
\lim _{h \rightarrow 0} \frac{1}{h} \int_{0}^{x} \frac{(x+h-y)^{t-1}-(x-y)^{t-1}}{\Gamma(t)} f(y) d y=J^{t-1} f(x)
$$

e

$$
\lim _{h \rightarrow 0} \frac{1}{h} \int_{x}^{x+h} \frac{(x+h-y)^{t-1}}{\Gamma(t)} f(y) d y=0,
$$

o resultado segue para $f \in C(0,1)$. Logo, o mesmo ocorrerá para funções em $C_{0}^{\infty}(0,1)$ e conseqüentemente para as funções em $L_{2}(0,1)$.

Lema 3.7 Dado $f \in L_{2}(0,1)$ definimos

$$
F(x)=\int_{0}^{x} \log (x-y) f(y) d y .
$$

$A$ integral converge absolutamente e $F(x) \rightarrow 0$ quando $x \rightarrow 0$.

Prova- A desigualdade

$$
\begin{aligned}
\left|\int_{0} \log (x-y) f(y) d y\right| & \leq \int_{0}^{r}|\log (x-y)||f(y)| d y \\
& \leq\left(\int_{0}^{r}|x-y|^{2} d y\right)^{1 / 2}\left(\int_{0}^{r}|f(y)|^{2} d y\right)^{1 / 2}
\end{aligned}
$$

nos astra que existe o limite

$$
\lim _{r \rightarrow x} \int_{0}^{r} \log (x-y) f(y) d y
$$

$|F(x)| \leq x^{3} / 3$ 
Lema 3.8 Para todo $\epsilon \in \mathbf{R} e \xi \in \mathbf{C}$ tal que $\Re(\xi)>1$ temos

$$
\int_{z}^{x} \frac{(x-y)^{\xi-2}}{\Gamma(\xi-1)}(y-z)^{\epsilon} d y=\frac{(x-z)^{\xi-1+\epsilon} \Gamma(1+\epsilon)}{\Gamma(\xi+\epsilon)} .
$$

Prova- Basta observarmos que

$$
\int_{z}^{x} \frac{(x-y)^{\xi-2}}{\Gamma(\xi-1)}(y-z)^{\epsilon} d y=\frac{(x-z)^{\xi-1+\epsilon}}{\Gamma(\xi-1)} \int_{0}^{1} s^{\xi-2}(1-s)^{\epsilon} d s .
$$

Teorema 3.6 Dado $f \in L_{2}(0,1)$ consideremos

$$
F(x)=\int_{0}^{x} \log (x-y) f(y) d y .
$$

\section{Então}

1. se $\omega \neq 0$ é um número complexo com $\Re(\omega) \geq 0$ e $\lim _{t \rightarrow 0} \frac{1}{t}\left(J^{\omega t} f-f\right)=g$ existe em $L_{2}(0,1)$, então $F \in H^{1}(0,1)$ e $g=\omega\left(F^{\prime}+\gamma f\right)$, onde $\gamma=-\Gamma^{\prime}(1)$.

2. se $F \in H^{1}(0,1)$, entâo $\lim _{t \rightarrow 0} \frac{1}{t}\left(J^{t} f-f\right)=F^{\prime}+\gamma f$ em $L_{2}(0,1)$.

Prova- 1. Para $\Re(\xi) \geq 0$, pelo Lema (3.6), temos que $J^{\xi+1} f(x)=\int_{0}^{x} J^{\xi} f(y) d y$. Portanto,

$$
\frac{1}{\Gamma(\xi+1)} \int_{0}^{x}(x-y)^{\xi} f(y) d y=\int_{0}^{x} J^{\xi} f(y) d y
$$

Tomando $\xi=\omega t, \operatorname{com} t>0$, temos

$$
\frac{1}{\Gamma(\omega t+1)} \int_{0}^{x}(x-y)^{\omega t} f(y) d y=\int_{0}^{x} J^{\omega t} f(y) d y .
$$

Multiplicando por $(\omega t)^{-1}$ e subtraindo $\frac{1}{\omega t} \int_{0}^{x} f(y) d y$ na igualdade acima concluímos

$$
\int_{0}^{x} \frac{1}{\omega t}\left(\frac{(x-y)^{\omega t}}{\Gamma(\omega t+1)}-1\right) f(y) d y=\int_{0}^{x} \frac{1}{\omega t}\left(J^{\omega t} f(y)-f(y)\right) d y .
$$

Fazendo $t \rightarrow 0$

$$
\begin{aligned}
\lim _{t \rightarrow 0} \int_{0}^{x} \frac{1}{\omega t}\left(\frac{(x-y)^{\omega t}}{\Gamma(\omega t+1)}-1\right) f(y) d y & =\int_{0}^{x} \lim _{t \rightarrow 0} \frac{1}{\omega t}\left(\frac{(x-y)^{\omega t}}{\Gamma(\omega t+1)}-1\right) f(y) d y \\
& =\int_{0}^{x}\left(\log (x-y)-\Gamma^{\prime}(1)\right) f(y) d y .
\end{aligned}
$$


Pela existência do $\lim _{t \rightarrow 0} \frac{1}{t}\left(J^{\omega t} f-f\right)=g$ em $L_{2}(0,1)$, otemos

$$
\lim _{t \rightarrow 0} \int_{0}^{x} \frac{1}{\omega t}\left(J^{\omega t} f(y)-f(y)\right) d y=\frac{1}{\omega} \int_{0}^{x} g(y) d y .
$$

Ou seja,

$$
F(x)=\int_{0}^{x}\left(\frac{g(y)}{\omega}-\gamma f(y)\right) d y .
$$

Portanto, $F$ é absolutamente contínua com derivada $F^{\prime}=\frac{1}{\omega} g-\gamma f$.

2. Seja $f \in L_{2}(0,1)$ tal que $f \in H^{1}(0,1)$. Podemos supor que $F(0)=0$. É fácil ver que $J^{1} F^{\prime}=F$ e portanto, $J^{\xi-1} F=J^{\xi} F^{\prime}$. Por outro lado, para $\Re(\xi)>1$ temos

$$
\begin{aligned}
J^{\xi} F^{\prime}(x) & =J^{\xi-1} F(x)=\int_{0}^{x} \int_{0}^{y} \frac{(x-y)^{\xi-2}}{\Gamma(\xi-1)} \log (y-z) f(z) d z d y \\
& =\int_{0}^{x}\left(\int_{z}^{x} \frac{(x-y)^{\xi-2}}{\Gamma(\xi-1)} \log (y-z) d y\right) f(z) d z
\end{aligned}
$$

Para determinarmos o valor de $\int_{z}^{x}(x-y)^{\xi-2} \log (y-z) / \Gamma(\xi-1) d y$ basta calcularmos a derivada com relação a $\epsilon$, em $\epsilon=0$, das funções do Lema (3.8). Obtemos então

$$
\int_{z}^{x} \frac{(x-y)^{\xi-2}}{\Gamma(\xi-1)} \log (y-z) d y=\frac{(x-z)^{\xi-1}}{\Gamma(\xi)}\left(\frac{\log (x-z)+\Gamma^{\prime}(1)}{\Gamma(1)}-\frac{\Gamma^{\prime}(\xi)}{\Gamma(\xi)}\right)
$$

Portanto,

$$
\begin{aligned}
J^{\xi} F^{\prime}(x) & =\int_{0}^{x} \frac{(x-z)^{\xi-1} \log (x-z) \Gamma(\xi)-(x-z)^{\xi-1} \Gamma^{\prime}(\xi)}{\Gamma^{2}(\xi)} f(z) d z \\
& +\Gamma^{\prime}(1) \int_{0}^{x} \frac{(x-z)^{\xi-1}}{\Gamma(\xi)} f(z) d z
\end{aligned}
$$

Ou seja,

$$
J^{\xi} F^{\prime}(x)=\frac{\partial}{\partial \xi} J^{\xi} f(x)-\gamma J^{\xi} f(x) \text { para todo } x \in(0,1) .
$$

Assim, para $\Re(\alpha)>1$ e $\Re(\beta)>1$, temos

$$
J^{\beta} f-J^{\alpha} f=\int_{\alpha}^{\beta}\left(J^{\xi} F^{\prime}+\gamma J^{\xi} f\right) d \xi .
$$

Esta igualdade vale também para $\Re(\alpha) \geq 0$ e $\Re(\beta) \geq 0$. Fazendo $\alpha \rightarrow 0$, temos

$$
\frac{1}{\beta}\left(J^{\beta} f-f\right)=\frac{1}{\beta} \int_{0}^{\beta} J^{\xi}\left(F^{\prime}+\gamma f\right) d \xi
$$


Como

$$
\left\|\frac{1}{\beta}\left(J^{\beta} f-f\right)-\left(F^{\prime}+\gamma f\right)\right\|_{2} \leq \frac{1}{\beta} \int_{0}^{\beta}\left\|J^{\xi}\left(F^{*}+\gamma f\right)-\left(F^{\prime}+\gamma f\right)\right\|_{2} d \xi
$$

chegamos ao que queríamos.

Considerando $J^{t}$ como um semigrupo em $\Re(t) \geq 0$, ou $t \geq 0$, ou sobre qualquer outro raio ou setor no semiplano direito, obtemos o mesmo gerador $A$ :

$$
\mathcal{D}(A)=\left\{f \in L_{2}(0,1): F \in H^{1}(0,1)\right\}
$$

e para $f \in \mathcal{D}(A)$, temos

$$
A f=F^{\prime}+\gamma f
$$

Consideremos o $C_{0}$-grupo $J^{i t}, t \in \mathbf{R}$, de operadores lineares limitados sobre $L_{2}(0,1)$. A partir dele estudaremos o comportamento do espectro do grupo e o espectro do seu gerador infinitesimal.

Lema 3.9 Para qualquer número complexo $p \operatorname{com} \Re(p)>0$, a funçâo

$$
g_{p}(x)=x^{p} \sqrt{2 \Re(p)+1}
$$

satisfaz: $g_{p} \in L_{2}(0,1),\left\|g_{p}\right\|_{2}=1 e J^{i t} g_{p}(x)=x^{i t} g_{p}(x) \Gamma(p+1) / \Gamma(p+1+i t)$.

Prova- As duas primeiras afirmativas são de imediatas. A terceira segue do fato que

$$
J^{i t} g_{p}(x)=\frac{\sqrt{2 \Re(p)+1}}{\Gamma(i t)} x^{i t+p} \int_{0}^{1} s^{p}(1-s)^{i t-1} d s \text {. }
$$

Teorema 3.7 Para $t \neq 0$, temos

$$
\sigma\left(J^{i t}\right)=\left\{z \in \mathbf{C}: e^{-|t| \pi / 2} \leq|z| \leq e^{|t| \pi / 2}\right\} .
$$

Prova- $O C_{0}$-grupo $J^{i t}, t \in \mathbf{R}$, satisfaz

$$
\left\|J^{i t}\right\| \leq \frac{e^{\pi|\Im(t)| / 2}}{(1-2 \Re(t))}=e^{|t| \pi / 2} .
$$

Para cada $t \in \mathbf{R}, J^{i t}$ é um operador linear limitado. Logo, $\sigma\left(J^{i t}\right) \neq \emptyset$ e obtemos a seguinte aproximação para o raio espectral:

$$
r\left(J^{i t}\right) \leq e^{|t| \pi / 2}
$$


Pelo fato de $\left(J^{i t}\right)^{-1}=J^{-i t}$ segue que

$$
\sigma\left(J^{i t}\right) \subset\left\{z \in \mathrm{C}: e^{-|t| \pi / 2} \leq|z| \leq e^{|t| \pi / 2}\right\} .
$$

Mostremos que, se $z \in \mathbf{C}$ é tal que $e^{-|t| \pi / 2}<|z|<e^{|t| \pi / 2}$, então $z \in \sigma\left(J^{i t}\right)$. Sendo o espectro um conjunto fechado temos a igualdade desejada para $t \neq 0$. Seja $z$ nas condições acima. Definamos.

$$
p_{n}=R_{n} e^{i \theta}=e^{\alpha+\frac{(2 \pi n)}{|t|}} e^{i \theta}
$$

onde $\alpha=1-(\arg z) / t$ e $\theta=(\log |z|) / t$. Logo, temos que $g_{p_{n}} \in L_{2}(0,1)$ e $\left\|g_{p_{n}}\right\|_{2}=1$, para todo $n \in N$. Ainda,

$$
J^{i t} g_{p_{n}}(x)-\frac{\Gamma\left(p_{n}+1\right)}{\Gamma\left(p_{n}+1+i t\right)} g_{p_{n}}(x)=\frac{\Gamma\left(p_{n}+1\right)}{\Gamma\left(p_{n}+1+i t\right)}\left(x^{i t}-1\right) g_{p_{n}}(x)
$$

Como

$$
\int_{0}^{1}\left|x^{i t}-1\right|^{2}\left|g_{p_{n}}(x)\right|^{2} d x=2-2 \frac{\left(2 \Re\left(p_{n}\right)+1\right)^{2}}{\left(2 \Re\left(p_{n}\right)+1\right)^{2}+t^{2}}
$$

e

$$
\begin{aligned}
\frac{\Gamma\left(p_{n}+1\right)}{\Gamma\left(p_{n}+1+i t\right)} & =\sqrt{\frac{p_{n}}{p_{n}+i t}}\left(\frac{p_{n}}{p_{n}+i t}\right)^{p_{n}} \frac{\left(1+O\left(\left|p_{n}\right|^{-1}\right)\right)}{\left(p_{n}+i t\right)^{i t} e^{-i t}\left(1+O\left(\left|p_{n}+i t\right|^{-1}\right)\right)} \\
& =p_{n}^{-i t} e^{i t}\left(1+O\left(\left|p_{n}\right|^{-1}\right)\right)=|z| e^{\text {argz }}\left(1+O\left(R_{n}^{-1}\right)\right)
\end{aligned}
$$

quando $n \rightarrow \infty$, temos que

$$
\left\|J^{i t} g_{p_{n}}-z g_{p_{n}}\right\|_{2} \leq\left\|J^{i t} g_{p_{n}}-\frac{\Gamma\left(p_{n}+1\right)}{\Gamma\left(p_{n}+1+i t\right)} g_{p_{n}}\right\|_{2}+|z| O\left(R_{N}^{-1}\right)
$$

quando $n \rightarrow \infty$. Ou seja,

$$
\left\|J^{i t} g_{p_{n}}-z g_{p_{n}}\right\|_{2} \rightarrow 0 \text { quando } n \rightarrow \infty .
$$

Portanto, $z \in \sigma\left(J^{i t}\right)$.

$\mathrm{Na}$ realidade demonstramos a existência de uma seqüência $g_{p_{n}}$ tal que $\left\|g_{p_{n}}\right\|_{2}=1$ e $\left\|J^{i t} g_{p_{n}}-z g_{p_{n}}\right\|_{2} \rightarrow 0$ quando $n \rightarrow \infty$. Uma pergunta que segue é: em que parte o espectro (pontual, residual, contínuo) está $z$ ? O número complexo $z$ nas condições acima pertence ao que chamamos espectro aproximante do operador que engloba o espectro pontual, o espectro contínuo e podendo ter parte do residual. Para maiores detalhes a respeito deste tipo de divisão do espectro ver [8]. Devido às inclusões espectrais temos que $z \in C \sigma\left(J^{i t}\right)$.

Passemos à análise de estimativas para $\|R(z, A)\|$, quando $z \in \rho(A)$. 
Proposição 3.5 Para todo $z \in \mathrm{C}$, temo: que

$$
R(z, A)=\int_{0}^{\infty} \epsilon^{-z t} J^{t} d t
$$

Prova- A função $z \rightarrow R(z, A)$ é inteira. Mostremos que o mesmo ocorre para a função $z \rightarrow \int_{0}^{\infty} e^{-z t} J^{t} d t$. Para tanto, basta mostrar que a integral existe para todo $z \in$ C. Para $\Re(z) \neq 0$, temos

$$
\begin{aligned}
\left\|\int_{r}^{s} e^{-z t} J^{t} d t\right\| & \leq \int_{r}^{s} e^{-\Re(z)} \frac{1}{t|\Gamma(t)|} d t \\
& \leq \frac{C}{(\Re(z))^{3 / 2}} \int_{\Re(z) r}^{\Re(z) s} e^{-\tau}(\tau)^{1 / 2} d \tau \rightarrow \frac{C}{(\Re(z))^{3 / 2}} \Gamma(3 / 2)
\end{aligned}
$$

quando $s \rightarrow \infty$ e $r \rightarrow 0$.

Se $\Re(z)=0$ temos

$$
\left\|\int_{r}^{s} e^{-z t} J^{t} d t\right\| \leq \int_{r}^{s} \frac{1}{t|\Gamma(t)|} d t=\int_{r}^{s} e^{-t} \frac{e^{t}}{|\Gamma(t+1)|} d t .
$$

Mas, $K=\max \left\{e^{t} /|\Gamma(t+1)|: t \geq 0\right\}<\infty$. Pois

$$
\frac{e^{k t}}{|\Gamma(t+1)|}=\frac{1}{\sqrt{2 \pi}} e^{(k+1-\log t) t} e^{-\log t / 2} \frac{1}{\left(1+O\left(|t|^{-1}\right)\right)}
$$

que converge para zero quando $t \rightarrow \infty$, e

$$
\frac{e^{k t}}{|\Gamma(t+1)|} \leq C e^{k i}(t+1)^{1 / 2} e^{\pi|\Im(t)| / 2}
$$

que converge para 1 quando $t \rightarrow 0$. Como

$$
R(z, A)=\int_{0}^{\infty} e^{-z t} J^{t} d t
$$

para $\Re(z)>\omega$, para algum $\omega \in \mathbf{R}$, pelo princípio da continuação analítica segue o resultado.

Assim, se $f \in L_{2}(0,1)$ temos

$$
\begin{aligned}
R(z, A) f(x) & =\int_{0}^{\infty} \int_{0}^{x} e^{-z t} \frac{(x-y)^{t-1}}{\Gamma(t)} f(y) d y d t \\
& =\int_{0}^{x} \int_{0}^{\infty} e^{-z t} \frac{(x-y)^{t-1}}{\Gamma(t)} f(y) d t d y
\end{aligned}
$$


Chamando

$$
E(u, z)=\int_{0}^{\infty} \frac{e^{-z t} u^{t-1}}{\Gamma(t)} d t \text { para } u>0
$$

temos

$$
R(z, A) f(x)=\int_{0}^{x} E(x-y, z) f(y) d y
$$

Repetindo argumentos semelhantes aos da Proposição (3.1) temos

$$
\|R(z, A)\| \leq \int_{0}^{1}|E(u, z)| d u .
$$

Proposição 3.6 Se $z=\xi+i \eta$, então $\|R(z, A)\|$ é uniformemente limitado quando $\xi \rightarrow \infty$.

Prova- Tomando $\xi \geq-1$, temos

$$
\begin{aligned}
\int_{0}^{1}|E(u, z)| d u & \leq \int_{0}^{\infty} \int_{0}^{1} \frac{e^{-\Re(z) t} u^{t-1}}{|\Gamma(t)|} d u d t \\
& =\int_{0}^{\infty} \frac{e^{-\Re(z) t}}{t|\Gamma(t)|} d t=\int_{0}^{\infty} e^{-(\Re(z)+2) t} \frac{e^{2 t}}{|\Gamma(t+1)|} d t
\end{aligned}
$$

Chamando $K=\max \left\{e^{2 t} /|\Gamma(t+1)|: t \geq 0\right\}$, obtemos

$$
\int_{0}^{1}|E(u, z)| d u \leq \frac{K}{\Re(z)+3} \leq \frac{K}{2} .
$$

Lema 3.10 Se $z=\xi+i \eta \operatorname{com} \eta>\pi / 2$, entâo

$$
E(u, z)=\frac{-i}{u} \int_{0}^{\infty} e^{(\xi-\log u) i \tau} \frac{e^{-\tau \eta}}{\Gamma(-i \tau)} d \tau
$$

Prova- Segue de modo análogo à prova do Lema (3.5).

Lema 3.11 Seja $\tau>0$. Então

$$
|\Gamma(-i \tau)|=\frac{\sqrt{2 \pi} e^{-\tau \pi / 2}}{\sqrt{\tau-\tau e^{-\pi / \tau}}} .
$$

Lema 3.12 Seja $\eta>\pi / 2$. A função $\tau \rightarrow e^{-\tau \eta} / \Gamma(-i \tau)$, juntamente com suas derivadas, tendem a zero quando $\tau \rightarrow \infty$. 
Proposição 3.7 Se $z=\xi+i \eta$. com $\eta>\pi / 2$, então $\|R(z, A)\|$ é uniformemente limitado sobre $-\infty<\xi<\infty$.

Prova- Pelo Lema (3.10) segue que

$$
\begin{aligned}
E(u, z) & =\frac{-i}{u} \int_{0}^{\infty} e^{(\xi-\log u) i \tau} \frac{\epsilon^{-\tau \eta}}{\Gamma(-i \tau)} d \tau \\
& =\frac{-1}{u(\xi-\log u)} \int_{0}^{\infty} e^{i \tau(\xi-\log u)} \frac{\partial}{\partial \tau}\left(\frac{e^{-\tau \eta}}{\Gamma(-i \tau)}\right) d \tau
\end{aligned}
$$

Onde a última igualdade decorre de

$$
\frac{e^{-\tau \eta} e^{i \tau(\xi-\log u)}}{i(\xi-\log u) \Gamma(-i \tau)} \rightarrow 0 \text { quando } \tau \rightarrow 0 \text { e } \tau \rightarrow \infty
$$

Integrando por partes novamente temos

$$
E(u, z)=\frac{1}{u(\xi-\log u)^{2}}\left(i+i \int_{0}^{\infty} e^{i \tau(\xi-\log u)} \frac{\partial^{2}}{\partial \tau^{2}}\left(\frac{e^{-\tau \eta}}{\Gamma(-i \tau)}\right) d \tau\right)
$$

Para $|\xi-\log u| \geq 1$ a seguinte limitação para $|E(u, z)|$ é obtida utilizando a última igualdade

$$
|E(u, z)| \leq \frac{C}{u|\xi-\log u|^{2}}
$$

onde $C=1+\int_{0}^{\infty}\left|\partial^{2} / \partial \tau^{2}\left(e^{-\tau \eta} / \Gamma(-i \tau)\right)\right| d \tau$. Para $|\xi-\log u| \leq 1$ utilizando a represeutação obtida no Lema (3.10) obtemos

$$
|E(u, z)| \leq \frac{C_{1}}{u}
$$

onde $C_{1}=\int_{0}^{\infty}\left|e^{-\tau \eta} / \Gamma(-i \tau)\right| d \tau$. Assim, para $\xi \leq-1$ temos

$$
\|R(z, A)\| \leq \int_{0}^{e^{\xi-1}}|E(u, z)| d u+\int_{e^{\xi+1}}^{e^{\xi-1}}|E(u, z)| d u+\int_{e^{\xi+1}}^{1}|E(u, z)| d u .
$$

Como,

$$
\begin{aligned}
\int_{0}^{e^{\xi-1}}|E(u, z)| d u & \leq \int_{0}^{e^{\xi-1}} \frac{C}{u(\xi-\log u)^{2}} d u=C \\
\int_{e^{\xi-1}}^{e^{\xi+1}}|E(u, z)| d u & \leq \int_{e^{\xi-1}}^{e^{\xi+1}} \frac{C_{1}}{u} d u=2 C_{1} \mathrm{e} \\
\int_{e^{\xi+1}}^{1}|E(u, z)| d u & \leq \int_{e^{\xi+1}}^{1} \frac{C}{u(\xi-\log u)^{2}} d u=C(1 / \xi+1)
\end{aligned}
$$


temos que

$$
\|R(z, A)\| \leq 2\left(C+C_{1}\right)
$$

onde as constantes dependem somente de $\eta$. Como para o caso $\xi \geq-1$ já encontramos uma limitação independente de $\eta$, concluímos a demonstração.

Pelo fato de $\overline{E(u, z)}=E(u, \bar{z})$ temos que o resultado da proposição anterior também é válido para o caso $\eta<-\pi / 2$.

Proposição 3.8 Se $z=\xi+i \eta$, com $-\pi / 2<\eta<\pi / 2$, então $\|R(z, A)\|$ é ilimitado quando $\xi \rightarrow-\infty$.

Prova- Seja $p=e^{-(\xi+i \eta)}$. Então $\arg (p)=-\eta \in(-\pi / 2, \pi / 2),|p| \rightarrow \infty \mathrm{e}$ $\Re(p) \rightarrow \infty$ quando $\xi \rightarrow-\infty$. Consideremos

$$
g_{p}(x)=x^{p} \sqrt{2 \Re(p)+1} .
$$

Pelo Lema (3.9) temos

$$
\left\|g_{p}\right\|_{2}=1 \text { e } J^{t} g_{p}(x)=\frac{\Gamma(p+1)}{\Gamma(p+1+t)} x^{t} g_{p}(x) .
$$

Como $g_{p} \in \mathcal{D}(A)$, então

$$
\begin{aligned}
A g_{p}(x) & =\lim _{t \rightarrow 0} \frac{J^{t} g_{p}(x)-g_{p}(x)}{t}=\lim _{t \rightarrow 0} g_{p}(x)\left(\frac{\Gamma(p+1)}{\Gamma(p+1+t)} x^{t}-1\right) \\
& =g_{p}(x)\left(\log x-\frac{\Gamma^{\prime}(p+1)}{\Gamma(p+1)}\right) .
\end{aligned}
$$

As igualdades

$$
\int_{0}^{1}\left|g_{p}(x) \log x\right|^{2} d x=\frac{2}{2 \Re(p)+1}
$$

e

$$
\frac{\Gamma^{\prime}(p+1)}{\Gamma(p+1)}=\log p+O\left(|p|^{-1}\right) \text { quando, }|p| \rightarrow \infty,
$$

nos permitem concluir que

$$
\begin{aligned}
\left\|A g_{p}-z g_{p}\right\|_{2} & \leq\left\|g_{p} \log (\cdot)\right\|_{2}+\left|\frac{\Gamma^{\prime}(p+1)}{\Gamma(p+1)}+z\right| \\
& =\left\|g_{p} \log (\cdot)\right\|_{2}+O\left(|p|^{-1}\right),
\end{aligned}
$$

que tende a zero quando $\xi \rightarrow-\infty$. Como

$$
\|R(z, A)\| \geq \frac{\left\|g_{p}\right\|_{2}}{\left\|A g_{p}-z g_{p}\right\|_{2}},
$$


concluímos que $\|R(z, A)\| \rightarrow \infty$ quando $\xi \rightarrow-\infty$.

Desde que $\rho(i A)=i \rho(A)$ podemos reformular os resultados encontrados para estimar $\|R(z, i A)\|$.

Corolário 3.3 Maniendo as notaçôes anteriores temos:

1. $\|R(z, i A)\|$ é uniformemente limitado quando $\Im(z) \rightarrow \infty$.

2. Se $\Re(z)>\pi / 2$ ou $\Re(z)<-\pi / 2$, então $\|R(z, i A)\|$ é uniformemente limitado sobre $\Im(z) \in(-\infty, \infty)$.

3. Se $-\pi / 2<\Re(z)<\pi / 2$, então $\|R(z, i A)\|$ é ilimitado quando $\Im(z) \rightarrow-\infty$.

\subsection{Um contra exemplo em Espaços de Banach}

Uma tentativa natural, após o estudo da Teoria Espectral para espaços de Hilbert, seria obter uma versão do Teorema (3.3) para espaços de Banach. O exemplo a seguir mostra que isto não é possivel com as mesmas hipóteses.

Dados os espaços de Banach

$$
C_{0}\left(\mathbf{R}_{+}\right)=\left\{f: \mathbf{R}_{+} \rightarrow \mathbf{C} ; f \text { contínua e } \lim _{x \rightarrow \infty}|f(x)|=0\right\}
$$

e

$$
L_{1}\left(\mathbf{R}_{+}, e^{x} d x\right)=\left\{f: \mathbf{R}_{+} \rightarrow \mathbf{C}_{;} \int_{0}^{\infty}|f(x)| e^{x} d x<\infty\right\}
$$

consideremos o espaço de Banach

$$
E=C_{0}\left(\mathbf{R}_{+}\right) \cap L_{1}\left(\mathbf{R}_{+}, e^{x} d x\right)
$$

munido da norma

$$
\|f\|=\|f\|_{\infty}+\|f\|_{1}=\sup \left\{|f(x)|: x \in \mathbf{R}_{+}\right\}+\int_{0}^{\infty}|f(x)| e^{x} d x .
$$

Sobre $E$ consideremos a família de operadores lineares

$$
T(t) f(x)=f(x+t) \text { para } t \geq 0 \text {. }
$$


Teorema 3.8 A familia $T(t), t \geq 0$, é um $C_{0}$-semigrupo sobre $E$. O gerador infinitesimal é dado por $A f=f^{\prime}$ se $f \in \mathcal{D}(A)$, onde $\mathcal{D}(A)=\{f \in E$ : $f$ é continuamente diferenciável e $\left.f^{\prime} \in E\right\}$.

Prova- Primeiramente observemos que $T(t) f(x)=f(x+t)$ para $t \geq 0$ é uma família de operadores lineares limitados sobre $E$. Isto segue de

$$
\|T(t) f\| \leq\|f\|_{\infty}+e^{-t}\|f\|_{1} \leq\|f\| .
$$

Ainda mais, tomando para cada $\epsilon>0, f_{\epsilon} \in E$ definido por

$$
f_{\epsilon}(x)=\left\{\begin{array}{clrl}
0 & \text { se } & 0 \leq x \leq 2 t-2 \epsilon \\
(x-2 t+2 \epsilon) / 2 \epsilon & \text { se } & 2 t-2 \epsilon \leq x \leq 2 t \\
1 & \text { se } & 2 t \leq x \leq 2 t+\epsilon \\
-(x-2 t-2 \epsilon) / \epsilon & \text { se } & 2 t+\epsilon \leq x \leq 2 t+2 \epsilon \\
0 & \text { se } & & x \geq 2 t+2 \epsilon
\end{array}\right.
$$

para cada $t \geq 0$, temos que $\|T(t)\|=1$.

É imediato que $T(t+s)=T(t) T(s)$ e $T(0)=I$. Da continuidade uniforme de $f$ em $\mathbf{R}_{+}$segue que $\|T(t) f-f\|_{\infty} \rightarrow 0$, quando $t \rightarrow 0^{+}$.

Ainda, fixando $x_{0} \in \mathbf{R}_{+}$tal que $\int_{x_{0}}^{\infty}|f(x)| e^{x} d x<\epsilon$ e $\int_{x_{0}+t}^{\infty}|f(x)| e^{x} d x<\epsilon$ temos que $\int_{0}^{\infty}|f(x+t)-f(x)| e^{x} d x<3 \epsilon$ desde que $t$ seja pequeno. Portanto, $T(t)$, $t \geq 0$, é um $C_{0}$-semigrupo.

Passemos à análise do gerador de $T(t)$. Se $f \in \mathcal{D}(A)$ então existe

$$
\lim _{t \rightarrow 0^{+}} \frac{T(t) f-f}{t}=g \in E .
$$

Concluímos que a derivada à direita de $f$ existe e é contínua para todo $x \in \mathbf{R}_{+}$. Logo, $f$ é continuamente diferenciável e $f^{\prime} \in E$.

Reciprocamente, se $f \in C^{1}\left(\mathbf{R}_{+}\right)$com $f^{\prime} \in E$ por argumentos análogos aos a prova de que $T(t)$ é um $C_{0}$-semigrupo concluímos que $f \in \mathcal{D}(A)$.

Teorema 3.9 Com as notações do Teorema (3.8) temos

1. $P \sigma(A)=\{\lambda \in \mathrm{C}: \Re(\lambda)<-1\}$.

2. $\{\lambda \in \mathbf{C}: \Re(\lambda)>-1\} \subset \rho(A)$.

3. $s(A)=\sup \{\Re(\lambda): \lambda \in \sigma(A)\}=-1$.

4. $1 \in \sigma(T(1))$. 
Prova- Primeiramente determinemos o números complexos $\lambda$, tais que $f_{\lambda} \in \mathcal{D}(A)$ e $A f_{\lambda}=\lambda f_{\lambda}$. Três condições devem ser verificadas:

1. $e^{\lambda x} \rightarrow 0$ quando $x \rightarrow \infty$.

2. $\lambda e^{\lambda x} \rightarrow 0$ quando $x \rightarrow \infty$.

3. $\int_{0}^{\infty} e^{\Re(\lambda) x} e^{x} d x<\infty$.

Portanto, segue a primeira parte. Para a segunda afirmativa basta mostrarmos que

$$
\int_{0}^{\infty} e^{-\lambda s} T(s) f d s<\infty \text { se } \Re(\lambda)>-1,
$$

pois tomando $R(\lambda) f=\int_{0}^{\infty} e^{-\lambda s} T(s) f d s$ e repetindo argumentos análogos aos do Corolário (1.1) segue o resultado.

Como

$$
\left\|\int_{0}^{r} e^{-\lambda s} T(s) f d s\right\|_{\infty}=\sup \left\{\left|\int_{0}^{r} e^{-\lambda s} T(s) f(x) d s\right|: x \in \mathbf{R}_{+}\right\}
$$

e $\left|\int_{0}^{r} e^{-\lambda s} T(s) f(x) d s\right| \leq e^{-x}\|f\|_{1}$, temos que

$$
\left\|\int_{0}^{r} e^{-\lambda s} T(s) f d s\right\|_{\infty} \leq\|f\|_{1}
$$

ou seja,

$$
\left\|\int_{0}^{\infty} e^{-\lambda s} T(s) f d s\right\|_{\infty}<\infty
$$

Além disso,

$$
\begin{aligned}
\left\|\int_{0}^{r} e^{-\lambda s} T(s) f d s\right\|_{1} & \leq \int_{0}^{r} e^{-\Re(\lambda) s}\|T(s) f\|_{1} d s \\
& \leq \int_{0}^{r} e^{-\Re(\lambda) s} e^{-s}\|f\|_{1} d s=\frac{\|f\|_{1}}{-(\Re(\lambda)+1)}\left(e^{-(\Re(\lambda)+1) r}-1\right) .
\end{aligned}
$$

Portanto,

$$
\left\|\int_{0}^{\infty} e^{-\lambda s} T(s) f d s\right\|_{1}<\infty
$$

Finalmente, pelo fato de $T(t)$ ser operador positivo, para todo $t \geq 0$, ver [8] ou H. H. Schaefer, Banach lattices and Positives Operators, concluímos, em particular, que $1 \in \sigma(T(1))$, pois $r(T(t))=1$ para todo $t \geq 0$, o que prova 4 .

Observemos que $2 \pi i n \in \rho(A)$, para todo $n \in \mathrm{Z}$ e

$$
\|R(2 \pi i n, A) f\|=\|R(2 \pi i n, A) f\|_{\infty}+\|R(2 \pi i n, A) f\|_{1} \leq 2\|f\|_{1} \leq 2\|f\|_{.}
$$

Ou seja, encontramos um $C_{0}$-semigupo definido sobre um espaço de Banach, tal que 
1. $2 \pi i n \in \rho(A)$ para todo $n \in \mathbf{Z}$.

2. $\|R(2 \pi i n, A)\|$ é uniformemente limitado, para $n \in \mathbf{Z}$.

e, por outro lado, $1 \in \sigma(T(1))$. 


\section{Bibliografia}

[1] G. Bachman e L. Narici, Functional analysis, Academic Press, New York, 1966.

[2] J. B. Conway, Functions of One Complex Variable, Springer-Verlag, New York, 1978.

[3] L. Gearhart, Spectral theory for contraction semigroups on Hilbert spaces, Trans. Amer. Math. Soc. 236 (1978), 385-394.

[4] D. B. Henry, Evolutions Equations on Banach Spaces, Notas não publicadas do IME-USP, 1981.

[5] D. B. Henry, Topics in Analysis, Pub. Mat. Universitat Autònoma de Barcelona, 31 (1987).

[6] I. Herbst, The Spectrum of Hilbert Space Semigroups, J. Operator Theory 10 (1983), 87-94.

[7] E. Hille e R. S. Phillips, Functional analysis and semigroups, Amer. Math. Soc. Colloq. Publ., vol. 31, Amer. Math. Soc., Providence, R.I, 1957.

[8] Nagel (Ed), One-parameter Semigroups of Positive Operators, SpringerVerlag, Berlin, 1986.

[9] A. Pazy, Semigroups of Linear Operators and Applications to Partial Differential Equations, Springer-Verlag, New-York, 1983.

[10] J. Prüss, On the spectrum of $C_{0}$-semigroups, Amer. Math. Soc. 284 (1984), $847-855$.

[11] A. E. Taylor, Introduction to Function Analysis, Wiley, New York, 1958. 